\title{
Ground states of integrable quantum liquids
}

\author{
J.M.P. Carmelo ${ }^{1,2}$ and N.M.R. Peres ${ }^{2}$ \\ ${ }^{1}$ Instituto de Ciencia de Materiales, C.S.I.C., Cantoblanco, E - 28949 Madrid, Spain \\ ${ }^{2}$ Department of Physics, University of Évora, Apartado 94, P - 7001 Évora codex, Portugal
}

(Received July 1994)

\begin{abstract}
Based on a recently introduced operator algebra for the description of a class of integrable quantum liquids we define the ground states for all canonical ensembles of these systems. We consider the particular case of the Hubbard chain in a magnetic field and chemical potential. The ground states of all canonical ensembles of the model can be generated by acting onto the electron vacuum (densities $n<1$ ) or hole vacuum (densities $n>1$ ), suitable pseudoparticle creation operators. We also evaluate the energy gaps of the non-lowest-weight states (non - LWS's) and nonhighest-weight states (non - HWS's) of the eta-spin and spin algebras relative to the corresponding ground states. For all sectors of parameter space and symmetries the exact ground state of the many-electron problem is in the pseudoparticle basis the non-interacting pseudoparticle ground state. This plays a central role in the pseudoparticle perturbation theory.
\end{abstract}

PACS numbers: $75.10 \mathrm{Lp}, 72.15 . \mathrm{Nj}, 05.30 . \mathrm{Fk}, 03.65 . \mathrm{Ca}$

Typeset Using REVTEX 


\section{INTRODUCTION}

In contrast to higher dimension, the many-electron one-dimensional problem is non perturbative. However, it also represents one of the few cases of many-body theory where there exist a number of exact solutions available. The most important class of such electronic systems are the integrable models solvable by the "Bethe ansatz" (BA) [王,2, 3, :4. Within these integrable "Luttinger-liquids" [5,6] the multicomponent models describing interacting fermions have been extensively investigated [2, 3, 1, 8, 9, 9, 10, 11, 12, In spite of the non-Fermi liquid behavior, these systems show in the sectors of parameter space of symmetry $U(1)$ a "Landau-liquid" character [9, 10, 11, 12, 13, 14, 15].

For some particular choices of canonical ensembles of the Hubbard chain [3] and other integrable models it has been assumed that the ground state corresponds to filling symmetrically around the origin the BA quantum numbers [3, 10,13, 14, 16, 17, 18]. Following this assumption, the pseudoparticle-operator algebra introduced in Refs. 11, 13, 14, revealed that, in contrast to the electronic basis, the pseudoparticle basis is perturbative. In particular, it was found that the natural reference state of the pseudoparticle perturbation theory is the many-electron ground state, which in the new basis is the non-interacting pseudoparticle ground state. This implies that this state plays a crucial role in the pseudoparticle perturbation theory.

In this paper we present a detailed study of the ground-state problem which confirms that the many-electron ground state corresponds to filling symmetrically around the origin the BA quantum numbers and that in the pseudoparticle basis it is the non-interacting pseudoparticle ground state. One of our goals is to deepen and generalize to all sectors of parameter space of the Hubbard chain in the presence of a magnetic field and chemical potential the study of that ground state. Our study refers directly to the canonical ensembles belonging to the four sectors of parameter space where the symmetry is $U(1) \otimes U(1)$ which correspond to electronic densities $0<n<1$ and $1<n<2$ and spin densities $-n<m<0$ and $-0<m<n$ (for $0<n<1$ ) and $-(2-n)<m<0$ and $0<m<(2-n$ ) (for 
$1<n<2)$. However, it also provides the results for the sectors of higher symmetry and, therefore, for all canonical ensembles of the model which correspond to electronic densities $0 \leq n \leq 2$ and spin densities $-n \leq m \leq n$ (for $0 \leq n \leq 1)$ and $-(2-n) \leq m \leq(2-n)$ (for $1 \leq n \leq 2)$.

Previous studies of the Hubbard chain have focused mainly on electronic densities $0 \leq$ $n \leq 1$ and spin densities $0 \leq m \leq n$. However, in spite of the fact that the symmetries of the model provide information for the values of the physical quantities at densities $1 \leq n \leq 2$ and spin densities $-(2-n) \leq m \leq(2-n)$ and densities $0 \leq n \leq 1$ and spin densities $-n \leq m \leq 0$ from the corresponding values at densities $0 \leq n \leq 1$ and spin densities $0 \leq m \leq n$, we show in this paper that from the point of view of the pseudoparticle operator algebra it is useful to consider explicitly all parameter-space sectors.

In the case of integrable models of simple Abelian $U(1)$ symmetry the elementary excitations are generated by a single type of pseudoparticles [19]. In this paper we find that the description of the low-energy excitations of non-Abelian integrable Hamiltonians involves a larger set of pseudoparticles. We show that the description of all gapless excitations branches of the four $U(1) \otimes U(1)$ sectors of parameter space of the Hubbard chain in a magnetic field and chemical potential involves eight types of pseudoparticles. The corresponding pseudoparticle algebra generates the low-energy Hamiltonian eigenstates from the electron vacuum (densities $0<n<1$ ) and hole vacuum (densities $1<n<2$ ), respectively. The related study of the low-energy physics of the sectors of parameter space of higher symmetry in terms of the above pseudoparticles will be presented elsewhere [20]. This study leads to the symmetry transformations of the eight types of pseudoparticles and reveals that the holons, antiholons, and two types of spinons of Ref. [21] are nothing but limiting cases of our general pseudoparticles.

In the pseudoparticle basis the ground state of the many-body problem is, for all canonical ensembles and corresponding symmetries, a "non-interacting" state, i.e., a simple Slater determinant of filled pseudoparticle levels. In addition, in the above sectors of symmetry $U(1) \otimes U(1)$ the elementary excitations simply correspond to pseudoparticle-pseudohole 
processes around this ground state.

The present study of the low-energy physics of the four sectors of symmetry $U(1) \otimes U(1)$ of the Hubbard chain in a magnetic field and chemical potential introduces the eight types of pseudoparticles needed for a deeper understanding and description of the gapless excitations

in the sectors of higher symmetry $S O(4), S U(2) \otimes U(1)$, and $U(1) \otimes S U(2)$. Therefore, the present study is a necessary step for the full characterization and understanding of the low-energy physics of the integrable non-Abelian many-electron quantum problems.

In Section II we generalize the perturbation theory introduced in Ref. [11,13] to all the $U(1) \otimes U(1)$ sectors of parameter space of the model. This requires the introduction of eight pseudoparticle branches.

In Sec. III we confirm that in the case of canonical ensembles belonging the sectors of parameter space of lowest symmetry $U(1) \otimes U(1)$ the ground state corresponds to filling symmetrically around the origin the BA quantum numbers.

In Section IV we show that in all canonical ensembles of the four $U(1) \otimes U(1)$ sectors of parameter space the non-LWS's and non-HWS's of the eta-spin and spin algebras have an energy gap relative to the corresponding ground state. In addition, we evaluate the smallest of these gaps.

Finally, in Sec. V we present the discussion and concluding remarks.

\section{PSEUDOPARTICLE BRANCHES OF THE FOUR $U(1) \otimes U(1)$ SECTORS}

Consider the Hamiltonian describing the Hubbard chain in a magnetic field $H$ and chemical potential $\mu$ 8, 10, 17, 18]:

$$
\hat{H}=\hat{H}_{S O(4)}+2 \mu \hat{\eta}_{z}+2 \mu_{0} H \hat{S}_{z},
$$

where

$$
\hat{H}_{S O(4)}=-t \sum_{j, \sigma}\left[c_{j \sigma}^{\dagger} c_{j+1 \sigma}+c_{j+1 \sigma}^{\dagger} c_{j \sigma}\right]+U \sum_{j}\left[c_{j \uparrow}^{\dagger} c_{j \uparrow}-1 / 2\right]\left[c_{j \downarrow}^{\dagger} c_{j \downarrow}-1 / 2\right]
$$


Here the operator $c_{j \sigma}^{\dagger}\left(c_{j \sigma}\right)$ creates (annihilates) one spin $\sigma$ electron at the site $j$ and $t, U, \mu$, $H$, and $\mu_{0}$ are the transfer integral, the onsite Coulomb interaction, the chemical potential, the magnetic field, and the Bohr magneton, respectively. The operators,

$$
\hat{\eta}_{z}=-\frac{1}{2}\left[N_{a}-\sum_{\sigma} \hat{N}_{\sigma}\right], \quad \hat{S}_{z}=-\frac{1}{2} \sum_{\sigma} \sigma \hat{N}_{\sigma},
$$

are the diagonal generators of the $S U(2)$ eta-spin and spin algebras, respectively [22,23]. $\sigma$ refers to the spin projections $\sigma=\uparrow, \downarrow$ when used as an operator or function index and is given by $\sigma= \pm 1$ otherwise. In Eq. (3) $\hat{N}_{\sigma}=\sum_{j} c_{j \sigma}^{\dagger} c_{j \sigma}$ is the number operator for $\sigma$ spin-projection electrons.

The model (1) - (2) describes an interacting quantum system of $N_{\uparrow}$ up-spin electrons and $N_{\downarrow}$ down-spin electrons on a chain of $N_{a}$ sites with lattice constant $a$. We use periodic boundary conditions and consider $N_{a}$ to be even and, when $N=N_{a}$ (half filling), both $N_{\uparrow}$ and $N_{\downarrow}$ to be odd. Henceforth we employ units such that $a=t=\mu_{0}=\hbar=1$. The Fermi momenta are given by $k_{F \sigma}=\pi n_{\sigma}$ and $k_{F}=\left[k_{F \uparrow}+k_{F \downarrow}\right] / 2=\pi n / 2$, where $n_{\sigma}=N_{\sigma} / N_{a}$ and $n=N / N_{a}$. The dimensionless onsite interaction is $u=U / 4 t$.

In the absence of the chemical-potential and magnetic-field terms the Hamiltonian (1) reduces to (2) and has $S O(4)=S U(2) \otimes S U(2) / Z_{2}$ symmetry [22,23, 24,25,26,27. Since $N_{a}$ is even, the operator $\hat{\eta}_{z}+\hat{S}_{z}$ (see Eq. (3)) has only integer eigenvalues and all half-odd integer representations of $S U(2) \otimes S U(2)$ are projected out [21,23]. The two $S U(2)$ algebras - eta spin and spin - have diagonal generators given by Eq. (3) and off-diagonal generators 21.23

$$
\hat{\eta}=\sum_{j}(-1)^{j} c_{j \uparrow} c_{j \downarrow}, \quad \hat{\eta}^{\dagger}=\sum_{j}(-1)^{j} c_{j \downarrow}^{\dagger} c_{j \uparrow}^{\dagger},
$$

and

$$
\hat{S}=\sum_{j} c_{j \uparrow}^{\dagger} c_{j \downarrow}, \quad \hat{S}^{\dagger}=\sum_{j} c_{j \downarrow}^{\dagger} c_{j \uparrow},
$$

respectively.

In the presence of both the magnetic field and chemical potential terms, the symmetry is reduced to $U(1) \otimes U(1)$, with $\hat{\eta}_{z}$ and $\hat{S}_{z}$ commuting with $\hat{H}$. The eigenvalues $\eta_{z}$ and $S_{z}$ 
are determined by the values of the conserved numbers, as shown by Eq. (3). According to these eigenvalues, the system has different symmetries as follows [10,13]: when $\eta_{z} \neq 0$ and $S_{z} \neq 0$ the symmetry is $U(1) \otimes U(1)$, for $\eta_{z}=0$ and $S_{z} \neq 0$ (and $\mu=0$ ) it is $S U(2) \otimes U(1)$, when $\eta_{z} \neq 0$ and $S_{z}=0$ it is $U(1) \otimes S U(2)$, and at $\eta_{z}=0$ and $S_{z}=0$ (and $\mu=0$ ) the symmetry is $S O(4)$. The $U(1) \otimes U(1)$ symmetry sectors always correspond to two non-zero eigenvalues of the diagonal generators, whereas in sectors of higher symmetry, one or both of these eigenvalues vanish. There are four $U(1) \otimes U(1)$ sectors of parameter space which correspond to $\eta_{z}<0$ and $S_{z}<0 ; \eta_{z}<0$ and $S_{z}>0 ; \eta_{z}>0$ and $S_{z}<0$; and $\eta_{z}>0$ and $S_{z}>0$. There are two $S U(2) \otimes U(1)[$ and $U(1) \otimes S U(2)]$ sectors of parameter space which correspond to $S_{z}<0$ and $S_{z}>0$ (and to $\eta_{z}<0$ and $\eta_{z}>0$ ). There is one $S O(4)$ sector of parameter space [which is the above $\eta_{z}=0$ (and $\mu=0$ ) and $S_{z}=0$ "point"].

We restrict our analysis to the four sectors of lowest symmetry $U(1) \otimes U(1)$. In this case we can define the parameters $l=\operatorname{sgn}\left(\eta_{z}\right) 1$ and $l^{\prime}=\operatorname{sgn}\left(S_{z}\right) 1$ which classify these four sectors: we denote each of them by $\left(l, l^{\prime}\right)$ sector. The $(-1,-1) ;(-1,1) ;(1,-1)$; and $(1,1)$ sectors refer to electronic densities and spin densities $0<n<1$ and $0<m<n$; $0<n<1$ and $-n<m<0 ; 1<n<2$ and $0<m<(2-n)$; and $1<n<2$ and $-(2-n)<m<0$, respectively.

In the $(-1,-1)$ sector the BA solution refers only to the LWS's of both the eta-spin and spin algebras. Note that the Hamiltonian eigenstates of the remaining three $\left(l, l^{\prime}\right)$ sectors can be generated by multiple application of the operators $\hat{\eta}^{\dagger}(4)$ and $\hat{S}^{\dagger}(5)$ to the LWS's of the $(-1,-1)$ sector [2,23]. We find below that in the sectors where $\eta_{z}>0$ and (or ) $S_{z}>0$ the ground states and low-energy Hamiltonian eigenstates are HWS's of the eta-spin and (or ) spin algebras. However, the generation of these HWS's from the corresponding LWS's with the same $\eta$ and $S$ values [and $\eta_{z}=-\eta_{z}$ and (or ) $S_{z}=-S_{z}$ ] represents a too complicated and indirect description of the low-energy physics of the $\eta_{z}>0$ and (or ) $S_{z}>0$ sectors of parameter space. Instead, in this paper we describe the low-energy physics of these sectors directly from the corresponding BA solutions. We emphasize that the set of LWS's and (or ) HWS's of each of the four $\left(l, l^{\prime}\right)$ sectors of symmetry $U(1) \otimes U(1)$ represent four alternative 
choices for starting states to construct a complete set of Hamiltonian eigenstates [see Ref. [23] where the LWS's of the $(-1,-1)$ sector are used as starting states].

Since in the $(1,-1)$ [and $(-1,1)]$ sector the low-energy physics is determined by HWS's [and LWS's] of the eta-spin algebra and LWS's [and HWS's] of the spin algebra, henceforth we refer the LWS's and (or ) HWS's of the four sectors as [LWS,LWS]'s [sector $(-1,-1)]$, [LWS,HWS]'s [sector $(-1,1)]$, [HWS,LWS]'s [sector $(1,-1)]$, and [HWS,HWS]'s [sector $(1,1)]$. (This notation refers to the order [eta-spin,spin].)

Let $\left|\eta_{z}, S_{z}\right\rangle$ be a regular BA Hamiltonian eigenstate corresponding to the canonical ensemble of eigenvalues $\eta_{z}$ and $S_{z}$. By regular BA Hamiltonian eigenstates we mean here these states to which the BA solution of any of the four $U(1) \otimes U(1)$ sectors directly refers. Such states can be [LWS,LWS]'s, [LWS,HWS]'s, [HWS,LWS]'s, or [HWS,HWS]'s according to the sector of parameter space. The BA solution for the sectors $(-1,1),(1,-1)$, and $(1,1)$ is obtained as for the usual sector $(-1,-1)$, except that instead of the equations

$$
\hat{\eta}\left|\eta_{z}, S_{z}\right\rangle=\hat{S}\left|\eta_{z}, S_{z}\right\rangle=0, \quad \text { for } \quad \eta_{z}<0, S_{z}<0
$$

which refer to [LWS,LWS]'s, the following equations have to be fulfilled

$$
\hat{\eta}\left|\eta_{z}, S_{z}\right\rangle=\hat{S}^{\dagger}\left|\eta_{z}, S_{z}\right\rangle=0, \quad \text { for } \quad \eta_{z}<0, S_{z}>0
$$

for the $(-1,1)$ sector where the regular BA Hamiltonian eigenstates are [LWS,HWS]'s;

$$
\hat{\eta}^{\dagger}\left|\eta_{z}, S_{z}\right\rangle=\hat{S}\left|\eta_{z}, S_{z}\right\rangle=0, \quad \text { for } \quad \eta_{z}>0, S_{z}<0
$$

for the $(1,-1)$ sector where the regular BA Hamiltonian eigenstates are [HWS,LWS]'s; and

$$
\hat{\eta}^{\dagger}\left|\eta_{z}, S_{z}\right\rangle=\hat{S}^{\dagger}\left|\eta_{z}, S_{z}\right\rangle=0, \quad \text { for } \quad \eta_{z}>0, S_{z}>0
$$

for the $(1,1)$ sector where the regular BA Hamiltonian eigenstates are [HWS,HWS]'s.

In References [11, [13, [14] the LWS's of the usual $(-1,-1)$ sector were devided into two types: the LWS's I, which refer to real BA rapidities; and the LWS's II. Some or all the rapidities which describe the latter states are complex and non real [13]. In the canonical 
ensembles of that $U(1) \otimes U(1)$ sector both the non-LWS's and the LWS's II have finite energy gaps relative to the ground state $[13$.

As in the case of the [LWS,LWS]'s of the $(-1,-1)$ sector, the [LWS,HWS]'s, [HWS,LWS]'s, and [HWS,HWS]'s can be devided into [LWS,HWS]'s I, [HWS,LWS]'s I, and [HWS,HWS]'s I, described by real rapidities, and [LWS,HWS]'s II, [HWS,LWS]'s II, and [HWS,HWS]'s II. Some or all the rapidites which describe the latter states are complex and non real. In the four $\left(l, l^{\prime}\right)$ sectors the non-LWS's and non-HWS's and the states II have an energy gap relative to the ground state, which is always a [LWS,LWS] I, [LWS,HWS] I, [HWS,LWS] I, or [HWS,HWS] I. In Sec. IV we will show that for the non-LWS's and non-HWS's, whereas the gaps of the states II will be evaluated in a following paper [28]. At energy ranges smaller than those gaps the Hilbert subspace spanned by the regular BA states I with common $\eta_{z}$ and $S_{z}$ eigenvalues coincides with the full Hilbert space of the quantum problem (spanned by states with these eigenvalues). The perturbation theory introduced in Refs. 11.13 for the $(-1,-1)$ sector is generalized here for all $\left(l, l^{\prime}\right)$ sectors and refers to that Hilbert space. It corresponds to energy scales smaller than the above gaps.

Henceforth, we will call state I any [LWS,LWS] I, [LWS,HWS] I, [HWS,LWS] I, or [HWS,HWS] I, and state II any [LWS,LWS] II, [LWS,HWS] II, [HWS,LWS] II, or [HWS,HWS] II. We note that a state I or a state II is always a regular BA Hamiltonian eigenstate and, therefore, is not, by definition, a non-LWS and (or ) non-HWS.

Let us generalize to the states I of all the $U(1) \otimes U(1)$ sectors the pseudoparticle-operator algebra of the $(-1,-1)$ sector. In each of the $\left(l, l^{\prime}\right)$ sectors we have two types of pseudoparticles. Combining the four sectors we have eight types of operators $b_{q \alpha\left(l, l^{\prime}\right)}^{\dagger}$ and $b_{q \alpha\left(l, l^{\prime}\right)}$ which obey the usual fermionic algebra

$$
\left\{b_{q \alpha\left(l, l^{\prime}\right)}^{\dagger}, b_{q^{\prime} \alpha^{\prime}\left(l^{\prime \prime}, l^{\prime \prime \prime}\right)}\right\}=\delta_{q, q^{\prime}} \delta_{\alpha, \alpha^{\prime}} \delta_{l, l^{\prime \prime}} \delta_{l^{\prime}, l^{\prime \prime \prime}}
$$

and

$$
\left\{b_{q \alpha\left(l, l^{\prime}\right)}^{\dagger}, b_{q^{\prime} \alpha^{\prime}\left(l^{\prime \prime}, l^{\prime \prime \prime}\right)}^{\dagger}\right\}=0, \quad\left\{b_{q \alpha\left(l, l^{\prime}\right)}, b_{q^{\prime} \alpha^{\prime}\left(l^{\prime \prime}, l^{\prime \prime \prime}\right)}\right\}=0
$$


Therefore, there are eight quantum numbers or "colors" $\alpha\left(l, l^{\prime}\right)$, which are $c(-1,-1)$, $c(1,-1), c(-1,1), c(1,1), s(-1,-1), s(1,-1), s(-1,1)$, and $s(1,1)$. These correspond to eight different branches of $\alpha\left(l, l^{\prime}\right)$ pseudoparticles.

In each $\left(l, l^{\prime}\right) U(1) \otimes U(1)$ sector only the corresponding $\alpha\left(l, l^{\prime}\right)$ pseudoparticles participate in the construction of the states I, as we discuss below. Therefore, the algebra of Eqs. (10) and (11) may be replaced by $\left\{b_{q \alpha\left(l, l^{\prime}\right)}^{\dagger}, b_{q^{\prime} \alpha^{\prime}\left(l, l^{\prime}\right)}\right\}=\delta_{q, q^{\prime}} \delta_{\alpha, \alpha^{\prime}}$ and $\left\{b_{q \alpha\left(l, l^{\prime}\right)}^{\dagger}, b_{q^{\prime} \alpha^{\prime}\left(l, l^{\prime}\right)}^{\dagger}\right\}=0$, respectively. The corresponding discrete pseudomomentum values are

$$
q_{j}=\frac{2 \pi}{N_{a}} I_{j}^{\alpha\left(l, l^{\prime}\right)}
$$

where $I_{j}^{\alpha\left(l, l^{\prime}\right)}$ are consecutive integers or half integers. The representation (12) was used by Yang and Yang in the case of the one-dimensional boson gas with repulsive delta-function interaction [29].

There are $N_{\alpha\left(l, l^{\prime}\right)}^{*}$ possible $I_{j}^{\alpha\left(l, l^{\prime}\right)}$ values. A state I is specified by the distribution of $N_{\alpha\left(l, l^{\prime}\right)}$ occupied values, which we call $\alpha\left(l, l^{\prime}\right)$ pseudoparticles, over the $N_{\alpha\left(l, l^{\prime}\right)}^{*}$ available values.

Since only single and zero occupancy of the values $I_{j}^{\alpha\left(l, l^{\prime}\right)}$ are allowed, only pseudoparticles of the color $\alpha\left(l, l^{\prime}\right)$ can occupy the states labeled by the numbers $I_{j}^{\alpha\left(l, l^{\prime}\right)}$. Therefore, the pseudoparticles have a fermionic character, as assured by the anticommuting algebra (10) and (11). (The BA wave function vanishes for configurations showing other than single and zero occupancy of the values $I_{j}^{\alpha\left(l, l^{\prime}\right)}[\llbracket, 13$.$) . For the Hubbard model the BA spatial wave$ function for the states I depends on the quantum numbers $I_{j}^{\alpha\left(l, l^{\prime}\right)}$ through two sets of real numbers, which many authors call rapidities.

The BA solution for the model (1) can be obtained directly for all sectors of parameter space. In Appendix A we provide a short presentation of that solution in the case of the present four $\left(l, l^{\prime}\right)$ sectors of symmetry $U(1) \otimes U(1)$. In that Appendix we introduce the rapidity functions, two-pseudoparticle phase shifts, and some other quantities needed in this paper. Equations (A1) and (A2) define the above rapidites for a given choice of the distribution occupancy of the numbers $I_{j}^{\alpha\left(l, l^{\prime}\right)}$, i.e. for a given state I. (For the states II some or all rapidities are complex, non-real, numbers [28].) 
There are $N_{\alpha\left(l, l^{\prime}\right)}^{*}-N_{\alpha\left(l, l^{\prime}\right)}$ empty values, which we call $\alpha\left(l, l^{\prime}\right)$ pseudoholes. Carrying the BA solution in the same manner as for the usual $(-1,-1)$ sector we find the numbers $N_{\alpha\left(l, l^{\prime}\right)}^{*}$ and $N_{\alpha\left(l, l^{\prime}\right)}$ for the remaining three sectors. In Table 1 we give these numbers for the four sectors. These are conserving numbers involving the numbers of lattice sites $N_{a}$ and of $\sigma$ electrons $N_{\sigma}$.

The eta spin $\eta$ and spin $S$ values [and the corresponding eigenvalues of the diagonal generators (3), $\eta_{z}=l \eta$ and $\left.S_{z}=l^{\prime} S\right]$ can be expressed in terms of the numbers of pseudoholes as follows

$$
\eta=\frac{1}{2}\left[N_{c\left(l, l^{\prime}\right)}^{*}-N_{c\left(l, l^{\prime}\right)}\right]
$$

and

$$
S=\frac{1}{2}\left[N_{s\left(l, l^{\prime}\right)}^{*}-N_{s\left(l, l^{\prime}\right)}\right]=\frac{1}{2}\left[N_{c\left(l, l^{\prime}\right)}-2 N_{s\left(l, l^{\prime}\right)}\right]
$$

respectively. The numbers $I_{j}^{c\left(l, l^{\prime}\right)}$ are integers (or half integers) for $N_{s\left(l, l^{\prime}\right)}$ even (or odd), and $I_{j}^{s\left(l, l^{\prime}\right)}$ are integers (or half integers) for $N_{s\left(l, l^{\prime}\right)}^{*}$ odd (or even).

Let $|V ;-1, \mp 1\rangle$ and $|V ; 1, \mp 1\rangle$ be the vacuum states which correspond to taking the limit of the electronic density $n \rightarrow 0$ and $n \rightarrow 2$, respectively, keeping $\pm N_{\uparrow}> \pm N_{\downarrow}$. In $|V ;-1, \mp 1\rangle$ and $|V ; 1, \mp 1\rangle$ there are only holes and electrons, respectively.

Since the colors $\alpha\left(l, l^{\prime}\right)$ and the pseudomomentum $q$ are the only quantum numbers involved in the description of the pseudoparticles whose occupancy configurations define the states I, all the corresponding allowed configurations can be generated by applying to the corresponding vacuum $\left|V ; l, l^{\prime}\right\rangle$ the $\sum_{\alpha} N_{\alpha\left(l, l^{\prime}\right)}$ creation operators $b_{q \alpha\left(l, l^{\prime}\right)}^{\dagger}$, i.e.

$$
\left.\left.\left|\eta_{z}, S_{z}\right\rangle=\prod_{\alpha=c, s}\left[\prod_{q_{j}=q_{1}}^{q_{N_{\alpha}\left(l, l^{\prime}\right)}} b_{q \alpha\left(l, l^{\prime}\right.}^{\dagger}\right)\right] V ; l, l^{\prime}\right\rangle,
$$

[here $\left.l=\operatorname{sgn}\left(\eta_{z}\right), l^{\prime}=\operatorname{sgn}\left(S_{z}\right)\right]$ where the set of $q_{j}$ values, $q_{1}, q_{2}, \ldots, q_{\left.N_{\alpha\left(l, l^{\prime}\right.}\right)}$, refer to the $N_{\alpha\left(l, l^{\prime}\right)}$ occupied pseudomomenta out of the $N_{\alpha\left(l, l^{\prime}\right)}^{*}$ available pseudomomentum values.

In each canonical ensemble belonging the $\left(l, l^{\prime}\right)$ sector, out of the total of regular BA Hamiltonian eigenstates I and II, 


$$
\left[\begin{array}{c}
N_{a} \\
N_{s\left(l, l^{\prime}\right)}^{*}
\end{array}\right]\left[\left[\begin{array}{c}
N_{a} \\
N_{s\left(l, l^{\prime}\right)}
\end{array}\right]+\left[\begin{array}{c}
N_{a} \\
N_{s\left(l, l^{\prime}\right)}-2
\end{array}\right]\right]-\left[\left[\begin{array}{c}
N_{a} \\
N_{s\left(l, l^{\prime}\right)}^{*}+1
\end{array}\right]+\left[\begin{array}{c}
N_{a} \\
N_{s\left(l, l^{\prime}\right)}^{*}-1
\end{array}\right]\right]\left[\begin{array}{c}
N_{a} \\
N_{s\left(l, l^{\prime}\right)}-1
\end{array}\right]
$$

(this formula is a generalization of the $(-1,-1)$ result of Ref. [23]), there are

$$
\left[\begin{array}{c}
N_{a} \\
N_{s\left(l, l^{\prime}\right)}+N_{s\left(l, l^{\prime}\right)}^{*}
\end{array}\right]\left[\begin{array}{c}
N_{s\left(l, l^{\prime}\right)}^{*} \\
N_{s\left(l, l^{\prime}\right)}
\end{array}\right]
$$

Hamiltonian eigenstates I. (The square brackets in the above equation refer to the usual combinatoric coefficents.)

We emphasize that in the suitable limits formula (17) also applies to canonical ensembles of symmetry $S O(4), S U(2) \otimes U(1)$, and $U(1) \otimes S U(2)$. For instance, in the $n=1$ and $m=0$ $S O(4)$ canonical ensemble for any of the four choices of $\left(l, l^{\prime}\right)$ numbers Eq. (17) gives one. In this case these four choices are alternative representations of the same canonical ensemble, i.e. when $\eta_{z}=0$ (and $\mu=0$ ) and $S_{z}=0$ there is only one state I with $\eta=S=0$. This is both a LWS and a HWS of the eta-spin and spin algebras and is the $S O(4)$ ground state [20]. In this canonical ensemble there are neither LWS's I nor HWS's I excited singlet states of the eta-spin and (or ) spin algebras.

In each canonical ensemble of eigenvalues $\eta_{z}$ and $S_{z}$ the states I of the form (15) and of total number given by Eq. (17) constitute a complete orthonormal basis which spans an important Hilbert subspace, which we call $\mathcal{H}_{\mathcal{I}}$. At energy scales smaller than the gaps for non-LWS's, non-HWS's, and states II, $\mathcal{H}_{I}$ represents the full accessible Hilbert space. We emphasize that, in general, many states I in $\mathcal{H}_{I}$ have energies larger than these gaps. The low-energy physics is determined only by the states I whose energies are smaller than such gaps.

In Ref. [13], and following Refs. [3, 10, 11, 17, 18], it was assumed for the case of the sector $(-1,-1)$ that out of all corresponding states I of form (15), the ground state of eigenvalues $\eta_{z}$ and $S_{z}$ corresponds to filling symmetrically around the origin $N_{\alpha(-1,-1)}$ consecutive $I_{j}^{\alpha(-1,-1)}$ values of all colors $\alpha(-1,-1)$. In the present general case this leads to 


$$
\left|0 ; \eta_{z}, S_{z}\right\rangle=\prod_{\alpha=c, s}\left[\prod_{q=q_{F \alpha\left(l, l^{\prime}\right)}^{(-)}}^{q_{F \alpha\left(l, l^{\prime}\right)}^{(+)}} b_{q \alpha\left(l, l^{\prime}\right)}^{\dagger}\right]\left|V ; l, l^{\prime}\right\rangle
$$

where when $N_{\alpha\left(l, l^{\prime}\right)}$ is odd (or even) and $I_{j}^{\alpha\left(l, l^{\prime}\right)}$ are integers (or half integers) the pseudo-Fermi points are symmetric and read

$$
q_{F \alpha\left(l, l^{\prime}\right)}^{(+)}=-q_{F \alpha\left(l, l^{\prime}\right)}^{(-)}=\frac{\pi}{N_{a}}\left[N_{\alpha\left(l, l^{\prime}\right)}-1\right]
$$

If all pseudo-Fermi points are symmetric the state (18) has zero momentum and is nondegenerate. On the other hand, when at least one of the pseudoparticle or antipseudoparticle numbers $N_{\alpha\left(l, l^{\prime}\right)}$ is odd (or even) and $I_{j}^{\alpha\left(l, l^{\prime}\right)}$ are half integers (or integers) the corresponding pseudo-Fermi points are nonsymmetric and read either

$$
q_{F \alpha\left(l, l^{\prime}\right)}^{(+)}=\frac{\pi}{N_{a}} N_{\alpha\left(l, l^{\prime}\right)}, \quad-q_{F \alpha\left(l, l^{\prime}\right)}^{(-)}=\frac{\pi}{N_{a}}\left[N_{\alpha\left(l, l^{\prime}\right)}-2\right]
$$

or

$$
q_{F \alpha\left(l, l^{\prime}\right)}^{(+)}=\frac{\pi}{N_{a}}\left[N_{\alpha\left(l, l^{\prime}\right)}-2\right], \quad-q_{F \alpha\left(l, l^{\prime}\right)}^{(-)}=\frac{\pi}{N_{a}} N_{\alpha\left(l, l^{\prime}\right)}
$$

In this case the state (18) has finite momentum and is degenerate. Equivalent expressions can be obtained for the limits of the pseudo-Brioullin zones, $q_{\alpha\left(l, l^{\prime}\right)}^{( \pm)}$, if we replace $N_{\alpha\left(l, l^{\prime}\right)}$ by $N_{\alpha\left(l, l^{\prime}\right)}^{*}$ in Eqs. $(19)-(21)$. Except for terms of order $1 / N_{a}$, we have that $q_{F \alpha\left(l, l^{\prime}\right)}^{(+)}=$ $-q_{F \alpha\left(l, l^{\prime}\right)}^{(-)}=q_{F \alpha\left(l, l^{\prime}\right)}$ and $q_{\alpha\left(l, l^{\prime}\right)}^{(+)}=-q_{\alpha\left(l, l^{\prime}\right)}^{(-)}=q_{\alpha\left(l, l^{\prime}\right)}$, where

$$
q_{F \alpha\left(l, l^{\prime}\right)}=\frac{\pi N_{\alpha\left(l, l^{\prime}\right)}}{N_{a}}, \quad q_{\alpha\left(l, l^{\prime}\right)}=\frac{\pi N_{\alpha\left(l, l^{\prime}\right)}^{*}}{N_{a}}
$$

In some studies the full expressions (19) - (21) have to be used because the terms of order $1 / N_{a}$ play an important role. On the other hand, many quantities are in the thermodynamic limit insensitive to these $1 / N_{a}$ corrections and we can replace $q_{F \alpha\left(l, l^{\prime}\right)}^{( \pm)}$in many expressions by the pseudomomenta $\pm q_{F \alpha\left(l, l^{\prime}\right)}(22)$. In Table 1 we present the values of the pseudo-Fermi points and limits of the pseudo-Brillouin zones (22) for the four $\left(l, l^{\prime}\right)$ sectors.

In Sec. III we will confirm that the ground state of canonical ensembles of sectors of parameter space of symmetry $U(1) \otimes U(1)$ has the form (18). 
The Hamiltonian eigenstates (15), in number of (17) and of which the state (18) represents a particular choice, can be rewritten relatively to the latter state. In this case they correspond to pseudoparticle-pseudohole processes around the reference configuration (18) and read

$$
\left|\eta_{z}, S_{z}\right\rangle=\prod_{\alpha=c, s}\left[\prod_{i, j=1}^{N_{p h}^{\alpha\left(l, l^{\prime}\right)}} b_{q_{j} \alpha\left(l, l^{\prime}\right)}^{\dagger} b_{q_{i} \alpha\left(l, l^{\prime}\right)}\right]\left|0 ; \eta_{z}, S_{z}\right\rangle
$$

where $q_{j}\left(q_{i}\right)$ defines the different locations of the pseudoparticles (pseudoholes) relatively to $(18)$ and $N_{p h}^{\alpha\left(l, l^{\prime}\right)}$ is the number of $\alpha\left(l, l^{\prime}\right)$ pseudoparticle-pseudohole processes. When the starting ground state and the final state of an excitation I have different electronic numbers, $N_{\sigma}$, it can be decomposed in a ground-state - ground-state transition and a pseudoparticlepseudohole excitation around the end ground state [20].

All the Hamiltonian eigenstates (15) and (23) are states I. Non-LWS's and non-HWS's are generated by acting raising or lowering generators (4) and (5) on these states.

In the Hilbert subspace $\mathcal{H}_{I}$ spanned by the states I the Hubbard model can be written in the pseudoparticle basis. The derivation is as for the $(-1,-1)$ sector and the Hamiltoniamn (1) reads 13

$$
\begin{aligned}
\hat{H} & =\hat{H}_{S O(4)}+|\mu| \sum_{q}\left[1-\hat{N}_{c\left(l, l^{\prime}\right)}(q)\right]+\mu_{0}|H| \sum_{q}\left[1-\hat{N}_{s\left(l, l^{\prime}\right)}(q)\right] \\
& =\hat{H}_{S O(4)}+|\mu| \sum_{q}\left[1-\hat{N}_{c\left(l, l^{\prime}\right)}(q)\right]+\mu_{0}|H|\left[\sum_{q} \hat{N}_{c\left(l, l^{\prime}\right)}(q)-2 \sum_{q} \hat{N}_{s\left(l, l^{\prime}\right)}(q)\right],
\end{aligned}
$$

where the Hamiltonian $\hat{H}_{S O(4)}(2)$ is of the form

$$
\hat{H}_{S O(4)}=\sum_{q}\left[\hat{N}_{c\left(l, l^{\prime}\right)}(q)\left\{-2 t \cos \left[\hat{K}_{l, l^{\prime}}(q)\right]-U / 2\right\}+U / 4\right]
$$

$\hat{K}_{l, l^{\prime}}(q)$ is a rapidity operator [13] whose eigenvalues, $K_{l, l^{\prime}}(q)$, are studied in Appendix A [see Eqs. (A3) and (A4)], and $\hat{N}_{c\left(l, l^{\prime}\right)}(q)$ is the $c\left(l, l^{\prime}\right)$ pseudomomentum distribution operator. The operator $\hat{N}_{\alpha\left(l, l^{\prime}\right)}(q)$ has the form

$$
\hat{N}_{\alpha\left(l, l^{\prime}\right)}(q)=b_{q \alpha\left(l, l^{\prime}\right)}^{\dagger} b_{q \alpha\left(l, l^{\prime}\right)}
$$

and we can write the $\alpha\left(l, l^{\prime}\right)$ pseudoparticle number operator as 


$$
\hat{N}_{\alpha\left(l, l^{\prime}\right)}=\sum_{q} \hat{N}_{\alpha\left(l, l^{\prime}\right)}(q)
$$

It follows that the operators $\hat{\eta}_{z}$ and $\hat{S}_{z}$ read

$$
\hat{\eta}_{z}=l \sum_{q}\left[1-\hat{N}_{c\left(l, l^{\prime}\right)}(q)\right], \quad \hat{S}_{z}=l^{\prime} \sum_{q}\left[1-\hat{N}_{s\left(l, l^{\prime}\right)}(q)\right]
$$

This, together with the following relations valid for all $\left(l, l^{\prime}\right)$ sectors,

$$
-l \mu=|\mu| ; \quad-l^{\prime} H=|H|,
$$

justifies the form (24) of the Hamiltonian (1) in the pseudoparticle basis.

In normal order relatively to the state (18) the expression of this Hamiltonian involves the normal-ordered operators 13, 14

$$
: \hat{N}_{q \alpha\left(l, l^{\prime}\right)}:=: b_{q \alpha\left(l, l^{\prime}\right)}^{\dagger} b_{q \alpha\left(l, l^{\prime}\right)}:
$$

and has pseudoparticle forward-scattering terms only. (The expression of the normal-ordered operator : $b_{q \alpha\left(l, l^{\prime}\right)}^{\dagger} b_{q \alpha\left(l, l^{\prime}\right)}$ : of the right-hand side (rhs) of Eq. (30) is given in Eq. (43) below.) As in the case of the $(-1,-1)$ Hamiltonian of Ref. [13], it has an infinite number of terms which correspond to increasing scattering orders. To second order we find for each $\left(l, l^{\prime}\right)$ sector

$$
: \hat{H}:=\sum_{\alpha, q} \epsilon_{\alpha\left(l, l^{\prime}\right)}(q): \hat{N}_{q \alpha\left(l, l^{\prime}\right)}:+\frac{1}{2} \sum_{\alpha, q} \sum_{\alpha^{\prime}, q^{\prime}} f_{\alpha \alpha^{\prime}}^{l, l^{\prime}}\left(q, q^{\prime}\right): \hat{N}_{q \alpha\left(l, l^{\prime}\right)}:: \hat{N}_{q^{\prime} \alpha^{\prime}\left(l, l^{\prime}\right)}:+\ldots
$$

where the pseudoparticle bands $\epsilon_{\alpha\left(l, l^{\prime}\right)}(q)$ and the $f$ functions are evaluated as in the case of the $(-1,-1)$ sector 13,18 . Since the colors $\alpha\left(l, l^{\prime}\right)$ and $\alpha^{\prime}\left(l, l^{\prime}\right)$ of two-pseudoparticle quantities, such as the above $f$ functions and below phase shifts (see also Appendix A), refer to the same $\left(l, l^{\prime}\right)$ numbers, in order to simplify our notation we call in this case these colors $\alpha$ and $\alpha^{\prime}$, respectively. (The numbers $\left(l, l^{\prime}\right)$ need to appear only once in these functions.) The expressions of the bands are

$$
\epsilon_{c\left(l, l^{\prime}\right)}(q)=\epsilon_{c\left(l, l^{\prime}\right)}^{0}(q)+[|\mu|-U / 2]-\mu_{0}|H|,
$$

and 


$$
\epsilon_{s\left(l, l^{\prime}\right)}(q)=\epsilon_{s\left(l, l^{\prime}\right)}^{0}(q)+2 \mu_{0}|H|
$$

where the pseudoparticle bare spectra $\epsilon_{c\left(l, l^{\prime}\right)}^{0}(q)$ and $\epsilon_{s\left(l, l^{\prime}\right)}^{0}(p)$ are given by

$$
\epsilon_{c\left(l, l^{\prime}\right)}^{0}(q)=-2 t \cos K_{l, l^{\prime}}^{(0)}(q)+2 t \int_{-Q_{l, l^{\prime}}}^{Q_{l, l^{\prime}}} d k \widetilde{\Phi}_{c c}^{l, l^{\prime}}\left(k, K_{l, l^{\prime}}^{(0)}(q)\right) \sin k
$$

and

$$
\epsilon_{s\left(l, l^{\prime}\right)}^{0}(q)=2 t \int_{-Q_{l, l^{\prime}}}^{Q_{l, l^{\prime}}} d k \widetilde{\Phi}_{c s}^{l, l^{\prime}}\left(k, S_{l, l^{\prime}}^{(0)}(q)\right) \sin k
$$

respectively. The phase shifts $\widetilde{\Phi}_{\alpha \alpha^{\prime}}^{l, l^{\prime}}$ are defined in Eqs. (A39)-(A42) and the functions $K_{l, l^{\prime}}^{(0)}(q)$ and $S_{l, l^{\prime}}^{(0)}(q)$ are the solutions of Eqs. (A3)-(A4) for the reference state (18). The parameters $Q_{l, l^{\prime}}$ are given in Eq. (A22). The functions $K_{l, l^{\prime}}^{(0)}(q)$ and $S_{l, l^{\prime}}^{(0)}(q)$ can be defined in terms of the phase shifts (A39) and (A40), respectively, as follows

$$
K_{l, l^{\prime}}^{(0)}(q)=q-\int_{-Q_{l, l^{\prime}}}^{Q_{l, l^{\prime}}} d k \widetilde{\Phi}_{c c}^{l, l^{\prime}}\left(k, K_{l, l^{\prime}}^{(0)}(q)\right), \quad q=\int_{-Q_{l, l^{\prime}}}^{Q_{l, l^{\prime}}} d k \widetilde{\Phi}_{c s}^{l, l^{\prime}}\left(k, S_{l, l^{\prime}}^{(0)}(q)\right) .
$$

The pseudoparticle bands $\epsilon_{\alpha(-1,-1)}(q)=\epsilon_{\alpha}(q)$ are plotted in Figs. 7 and 8 of Ref. 17]. In the present $\left(l, l^{\prime}\right)$ sectors of parameter space of symmetry $U(1) \otimes U(1)$ the pseudoparticle energy spectra $\epsilon_{c\left(l, l^{\prime}\right)}(q)$ and $\epsilon_{s\left(l, l^{\prime}\right)}(q)$ defined by Eqs. (32) and (33) vanish at the pseudoFermi-points, i.e.,

$$
\epsilon_{\alpha\left(l, l^{\prime}\right)}\left(q_{F \alpha\left(l, l^{\prime}\right)}^{( \pm)}\right)=0
$$

The $f$ functions, $f_{\alpha \alpha^{\prime}}^{l, l^{\prime}}\left(q, q^{\prime}\right)$, of the rhs of Eq. (31) read

$$
\begin{aligned}
f_{\alpha \alpha^{\prime}}^{l, l^{\prime}}\left(q, q^{\prime}\right) & =2 \pi v_{\alpha\left(l, l^{\prime}\right)}(q) \Phi_{\alpha \alpha^{\prime}}^{l, l^{\prime}}\left(q, q^{\prime}\right)+2 \pi v_{\alpha^{\prime}\left(l, l^{\prime}\right)}\left(q^{\prime}\right) \Phi_{\alpha^{\prime} \alpha}^{l, l^{\prime}}\left(q^{\prime}, q\right) \\
& \left.\left.+\sum_{j= \pm 1} \sum_{\alpha^{\prime \prime}=c, s} 2 \pi v_{\alpha^{\prime \prime}\left(l, l^{\prime}\right)} \Phi_{\alpha^{\prime \prime} \alpha}^{l, l^{\prime}}\left(j q_{F \alpha^{\prime \prime}\left(l, l^{\prime}\right)}\right) q\right) \Phi_{\alpha^{\prime \prime} \alpha^{\prime}}^{l, l^{\prime}}\left(j q_{F \alpha^{\prime \prime}\left(l, l^{\prime}\right)}\right), q^{\prime}\right),
\end{aligned}
$$

where the two-pseudoparticle phase shifts $\Phi_{\alpha \alpha^{\prime}}^{l, l^{\prime}}\left(q, q^{\prime}\right)$ are defined by Eqs. (A18)-(A21) and the pseudoparticle group velocities are given by

$$
v_{\alpha\left(l, l^{\prime}\right)}(q)=\frac{d \epsilon_{\alpha\left(l, l^{\prime}\right)}(q)}{d q}
$$

The "light" velocities 


$$
v_{\alpha\left(l, l^{\prime}\right)} \equiv v_{\alpha\left(l, l^{\prime}\right)}\left(q_{F \alpha\left(l, l^{\prime}\right)}\right)
$$

play a determining role at the critical point and appear in the conformal-invariant expressions 8.,11,13]. (The velocities $v_{\alpha(-1,-1)}=v_{\alpha}$ are plotted in Fig. 9 of Ref. [17].)

Equation (31) is a generalization of the corresponding $(-1,-1)$ Hamiltonian of Ref. 13 for the three remaining sectors. We emphasize that in the present $U(1) \otimes U(1)$ sectors of parameter space and at energy scales smaller than the gaps for the non-LWS's, non-HWS's, and states II Eqs. (24), (25), and (31) refer to the expression of the full quantum-liquid Hamiltonian. In the electronic basis this is given by Eqs. (1) and (2). (In the case of the sector $(-1,-1)$, the pseudoparticle-operator representation (31) leads, in a natural way, to the low-energy spectrum studied in Refs. 8, 9, 10, 17, 18].)

Both the $f$ functions (38) and all the remaining higher order coefficients have universal forms in terms of the two-pseudoparticle phase shifts and pseudomomentum derivatives of the bands and their higher order "velocities". The two operators of the rhs of Eq. (31) are the Hamiltonian terms which are relevant at low energy 11.13.

The perturbative character of the pseudoparticle basis follows from the fact that, in contrast to the two-electron forward scattering amplitudes and vertices, the two-pseudoparticle $f$ functions (given by Eq. (38)) and the corresponding two-pseudoparticle forward-scattering amplitudes, which were calculated in Ref. [10] for the $(-1,-1)$ sector, do not diverge and are finite.

The combination of Eqs. (32), (33), and (37) allows the derivation of the density and magnetization curves which read

$$
\mu=-\left.l\left[\frac{U}{2}-\left.\epsilon_{c\left(l, l^{\prime}\right)}^{0}\left(q_{F c\left(l, l^{\prime}\right)}\right)\right|_{x}-\frac{1}{2} \epsilon_{s\left(l, l^{\prime}\right)}^{0}\left(q_{F s\left(l, l^{\prime}\right)}\right)\right]\right|_{x}
$$

and

$$
H=\left.l^{\prime}\left[\frac{\epsilon_{s\left(l, l^{\prime}\right)}^{0}\left(q_{F s\left(l, l^{\prime}\right)}\right)}{2 \mu_{0}}\right]\right|_{y},
$$

respectively, where, depending on the constraints imposed to the system, either $x=H$ or $x=m$ and either $y=\mu$ or $y=n$, respectively. 
The excited states I, which in the canonical ensembles of the four sectors of lowest symmetry are the only gapless states, never involve pseudoparticles with different $\left(l, l^{\prime}\right)$ numbers. In each of the four $\left(l, l^{\prime}\right)$ sectors of symmetry $U(1) \otimes U(1)$ there is a selection rule which states that out of the eight pseudoparticle branches only pseudoparticle-pseudohole transitions in each of the two corresponding bands $c\left(l, l^{\prime}\right)$ and $s\left(l, l^{\prime}\right)$ are allowed. Therefore, in the canonical ensembles of the $\left(l, l^{\prime}\right)$ sectors the states I (15), in number given by Eq. (17), refer to the two $c\left(l, l^{\prime}\right)$ and $s\left(l, l^{\prime}\right)$ bands only. This is also true for all states I associated with higher symmetry sectors [20].

\section{THE GROUND STATE IN THE $U(1) \otimes U(1)$ SECTORS}

In this section we use the pseudoparticle basis and the associated perturbative character of the quantum problem to justify that in canonical ensembles of the $\left(l, l^{\prime}\right)$ sectors the ground state has the form (18) and corresponds to filling symmetrically around the origin the BA quantum numbers.

To show that at given eigenvalues $\eta_{z}$ and $S_{z}$ and within all the states I of form (15) and in total number given by Eq. (17) the Hamiltonian eigenstate (18) (which is degenerate when its momentum is finite) is the state of minimal energy, we use the pseudoparticle basis of $\mathcal{H}_{I}$, where the Hamiltonian (1) and (2) is given by Eqs. (24) and (25), respectively.

In Appendix B we study the general energy expression for all states I of $\mathcal{H}_{I}$ (with common $\eta_{z}$ and $S_{z}$ eigenvalues), which is given by Eqs. (B1), (B2), and (B4). In the case of zero-spin density, $m=0$, the energy $E_{S O(4)}^{0}+(U / 2)\left[N-N_{a} / 2\right]$ was studied and plotted by Shiba [16] [here $E_{S O(4)}^{0}$ is the energy (B2) and (B4) of the sector $(-1,-1)$ ]. In that Appendix we devote particular attention to the states I of minimal and maximal energies. The study of the energy expressions for the different Hamiltonian eigenstates with common eigenvalues $\eta_{z}$ and $S_{z}$ identifies these two states I. The results are:

First, the study of the energy (B1) reveals that the energies of the states I of form (15) and with common eigenvalues $\eta_{z}$ and $S_{z}$ correspond to a continuous distribution without 
energy gaps.

Second, the minimal and maximal energies of that continuous distribution of energies of states with common eigenvalues $\eta_{z}$ and $S_{z}$ corresponds to the states I of pseudomomentum distributions $N_{\alpha\left(l, l^{\prime}\right)}(q)=N_{\alpha\left(l, l^{\prime}\right)}^{0}(q)$ and $N_{\alpha\left(l, l^{\prime}\right)}(q)=N_{\alpha\left(l, l^{\prime}\right)}^{*}(q)$, respectively, where $N_{\alpha\left(l, l^{\prime}\right)}^{0}(q)$ and $N_{\alpha\left(l, l^{\prime}\right)}^{*}(q)$ are given by Eqs. (B3) and (B5), respectively.

Third, since the energies of the states I of form (15) and with common eigenvalues $\eta_{z}$ and $S_{z}$ correspond to a continuous distribution without energy gaps, in order to show that (B11) and (B12) are the minimal and maximal values for these energies, respectively, it is enought to show that the energies $\left[E-E_{0}\right]$ and $\left[E-E_{*}\right]$ of the sub class of these states that can be generated from (18) and (B10), respectively, by changing the distribution occupancies of an arbitrary small density of pseudoparticles relative to the distributions (B3) and (B5), are positive and negative, respectively. Therefore, within all states (15) in number of (17) it is enough to evaluate the energies of the Hamiltonian eigenstates which differ from the reference distributions (B3) and (B5) by changing the occupied pseudomomenta by a small density for the two branches of $c\left(l, l^{\prime}\right)$ and $s\left(l, l^{\prime}\right)$ pseudoparticles.

Fourth, and in contrast to the electronic basis, we can use the perturbative character of the pseudoparticle basis [13] to expand the energies $\left[E-E_{0}\right] / N_{a}$ and $\left[E-E_{*}\right] / N_{a}$ of the above sub class of states in the densities of excited $\alpha\left(l, l^{\prime}\right)$ pseudoparticles, $n_{e x}^{\alpha\left(l, l^{\prime}\right)}$, relative to the reference distributions (B3) and (B5). Furthermore, we can write the Hamiltonian (1) with (2) given by (25) in normal order relative to the reference states (18) and (B10) [13]. In Sec. II we have presented the normal-ordered Hamiltonian relative to the state (18), Eq. (31). The normal-order character of these Hamiltonians implies that the corresponding energies are given relative to the states (18) and (B10), respectively. These Hamiltonians have an infinite number of terms [see Eq. (31) for the case of the state (18)] and, therefore, the evaluation of these energies seems to require the evaluation of an infinite number of energy contributions. However, the perturbative character of the pseudoparticle basis makes the problem much easier. This perturbative character rests on the fact that the evaluation of these energies up to the $i^{t h}$ order in these densities requires considering only the corre- 
sponding Hamiltonian terms of scattering orders less than or equal to $i$ [13]. This follows from the linearity of the density of excited $\alpha\left(l, l^{\prime}\right)$ pseudoparticles, which are the elementary "particles" of the quantum liquid, in $\delta N_{\alpha\left(l, l^{\prime}\right)}(q)=\left\langle\eta_{z}, S_{z}\left|: \hat{N}_{\alpha\left(l, l^{\prime}\right)}(q):\right| \eta_{z}, S_{z}\right\rangle$. Here, the normal-ordered pseudomomentum distribution relative to the states (18) and (B10) is given by

$$
: \hat{N}_{\alpha\left(l, l^{\prime}\right)}(q):=b_{q \alpha\left(l, l^{\prime}\right)}^{\dagger} b_{q \alpha\left(l, l^{\prime}\right)}-N_{\alpha\left(l, l^{\prime}\right)}^{0}(q)
$$

and

$$
: \hat{N}_{\alpha\left(l, l^{\prime}\right)}(q):=b_{q \alpha\left(l, l^{\prime}\right)}^{\dagger} b_{q \alpha\left(l, l^{\prime}\right)}-N_{\alpha\left(l, l^{\prime}\right)}^{*}(q)
$$

respectively.

Fifth, the perturbative character of the pseudoparticle basis together with the above analysis implies that in each canonical ensemble it is enough to consider the states called, in the case of a $(-1,-1)$ state (18), (B) and (C) in Refs. [11,13. These states can also be defined in the general case of the $\left(l, l^{\prime}\right)$ state (18) and of the state (B10) and correspond to a small density of pseudoparticle-pseudohole processes relative to the reference distributions (B3) and (B5). (The states (A) of Refs. [11,13] are associated with changes, $\Delta N_{\sigma}$, in the number of $\sigma$ electrons.) Moreover, one needs to evaluate the energies of these states up to second order in the density of $\alpha\left(l, l^{\prime}\right)$ pseudoparticles, $n_{e x}^{\alpha\left(l, l^{\prime}\right)}$, only. This involves only the oneand two-pseudoparticle terms of the corresponding normal-ordered Hamiltonian. [For the case of the state (18) see Eq. (31).] We find that for the reference states (18) and (B10) all such energies are positive and negative, respectively. (For the case of the $(-1,-1)$ reference state (18) these energies were evaluated in Ref. [13], and equal the energy spectrum studied in Ref. 8] when the number of both up-spin and down-spin electrons is kept constant.) This confirms that in each canonical ensemble of a $\left(l, l^{\prime}\right)$ sector (18) and (B10) are, within all states I of form (15), with common eigenvalues $\eta_{z}$ and $S_{z}$, and in number of (17), the Hamiltonian eigenstates of minimal and maximal energies, respectively. This implies that the energies $E$, Eq. (B1), of all remaining states I of that ensemble are such that 


$$
E_{0}<E<E_{*}
$$

where $E_{0}$ and $E_{*}$ are the energies (B11) and (B12), respectively. In addition, for each canonical ensemble of eigenvalues $\eta_{z}$ and $S_{z},\left[E_{*}-E_{0}\right]$ gives the energy width of the continuous distribution of energies corresponding to the whole set of $\left(l, l^{\prime}\right)$ states I of form (15).

We emphasize that only writing the Hamiltonian in the pseudoparticle-operator basis introduces the perturbative character of the quantum problem which simplified the above analysis. Here we have compared the energies of the states I only. Therefore, a complete proof of (18) being the Hamiltonian eigenstate of minimal energy in canonical ensembles of the present lowest-symmetry sectors of parameter space requires the evaluation of the energy gaps of the non-LWS's and non-HWS's and of the states II relative to that state, which have to be finite. While the gaps of the non-LWS's and non-HWS's are evaluated in Sec IV, the gaps of the states II are calculated elsewhere 28].

Finally, the study of the spectrum of the states II of the sectors of higher symmetry $S O(4), S U(2) \otimes U(1)$, and $U(1) \otimes S U(2)$ [20,28] reveals that in canonical ensembles of these sectors the ground state is also a state I of form (18), as we discuss in Ref. [20]. [When

$\eta_{z}=0$ and (or ) $S_{z}=0$, the $\alpha\left( \pm 1, l^{\prime}\right)$ and (or ) $\alpha(l, \pm 1)$ pseudoparticles correspond to alternative representations of the same ground state.] Therefore, in all canonical ensembles of the quantum problem and in the pseudoparticle basis the ground state is a simple Slater determinant of pseudoparticle levels of the universal form given by Eq. (18).

\section{ENERGY GAPS OF THE NON-LWS'S AND NON-HWS'S}

In Sec. III we have used the pseudoparticle basis to show that among all $\left(l, l^{\prime}\right)$ states I with common eigenvalues $\eta_{z}$ and $S_{z}$, in number of (17) and of the form (15), the Hamiltonian eigenstate (18) has minimal energy. Therefore, if in the corresponding canonical ensemble both all the non-LWS's and non-HWS's and all the states II (with common $\eta_{z}$ and $S_{z}$ eigenvalues) have a gap relative to the state (18), this state is the ground state. In this section we show that all such non-LWS's and non-HWS's have an energy gap and calculate 
the smallest of these gaps.

Let us consider the Hamiltonian $\hat{H}_{S O(4)}$, Eq. (2). This represents the Hubbard chain (1) at zero magnetic field and chemical potential and commutes with the six generators of Eqs. (3) - (5) and, therefore, has $S O(4)$ symmetry [23], as discussed in Sec. II.

Let $\left|\eta, S ; \eta_{z}, S_{z}\right\rangle$ be an arbitrary Hamiltonian eigenstate belonging the family of states with fixed eta spin and spin, $\eta$ and $S$, respectively. In $\left|\eta, S ; \eta_{z}, S_{z}\right\rangle \eta_{z}$ and $S_{z}$ are the eta-spin and spin projections, respectively, of this particular state. The whole family of states with the same values of eta spin $\eta$ and spin $S$ but different eta-spin and spin projections can be generated from one of the four [LWS,LWS], [LWS,HWS], [HWS,LWS], and [HWS,HWS] with these values of eta spin and spin. The [LWS,LWS] starting state, for example, is the state $|\eta, S ;-\eta,-S\rangle$ which belongs to the sector $(-1,-1)$. The remaing three choices for starting states are $|\eta, S ;-\eta, S\rangle,|\eta, S ; \eta,-S\rangle$, and $|\eta, S ; \eta, S\rangle$, respectively. Depending on the LWS or HWS character of the starting state, the family of states is generated by acting operators $\hat{\eta}$ and $\hat{S}$ or $\hat{\eta}^{\dagger}$ and $\hat{S}^{\dagger}$ of Eqs. (4) and (5) onto that state.

In Appendix $\mathrm{C}$ we consider fixed values of the chemical potential $\mu$ and magnetic field $H$ such that $\mu \neq 0$ and $H \neq 0$. The signs of the chemical potential and magnetic field fix the signs of $\eta_{z}$ and $S_{z}$ and choose the particular $\left(l, l^{\prime}\right)$ sector. According to Eq. (29), in the sectors $(-1,-1),(-1,1),(1,-1)$, and $(1,1)$ the chemical potential $\mu$ and magnetic field $H$ are such that $\mu>0$ and $H>0, \mu>0$ and $H<0, \mu<0$ and $H>0$, and $\mu<0$ and $H<0$, respectively. In that Appendix we show that at fixed and finite values of the chemical potential and magnetic field the lowest energy Hamiltonian eigenstate of a family of states $\left|\eta, S ; \eta_{z}, S_{z}\right\rangle$ with common values of $\eta$ and $S$ but different values of $\eta_{z}$ and $S_{z}$ is the [LWS,LWS], [LWS,HWS], [HWS,LWS], or [HWS,HWS] corresponding to the $\left(l, l^{\prime}\right)$ sector choosed by the signs of the chemical potential and magnetic field. We have also calculated the smallest energy gaps relative to that state, which are given in Eq. (C11).

The main goal of this section is, however, to show that within all states with different $\eta$ and $S$ values but the same eigenvalues $\eta_{z}$ and $S_{z}$, i.e. of states belonging the same canonical ensemble, the ground state (18) has minimal energy. 
Following the results of Sec. III, within all states I with the same eigenvalues $\eta_{z}$ and $S_{z}$ the state of minimal energy has the form (18). Therefore, we can restrict our considerations to the set of non-LWS's and non-HWS's, $\left|\eta, S ; \eta_{z}, S_{z}\right\rangle$, whose starting LWS's and (or) HWS's, $|\eta, S ; \pm \eta, \pm S\rangle$, are "ground states" of the form (18). Other non-LWS's and non-HWS's with the same $\eta_{z}$ and $S_{z}$ eigenvalues are of higher energy.

The non-LWS's and non-HWS's belong the same canonical ensemble and, therefore, have common $\eta_{z}$ and $S_{z}$ eigenvalues. We emphasize that their starting states, $|\eta, S ; \pm \eta, \pm S\rangle$, do not belong to that canonical ensemble. In the corresponding $\left(l, l^{\prime}\right)=\left(\operatorname{sgn}\left(\eta_{z}\right) 1, \operatorname{sgn}\left(S_{z}\right) 1\right)$ sector of symmetry $U(1) \otimes U(1)$, the energy of the ground state (18) of eigenvalues $\eta_{z}$ and $S_{z}$ can be written as

$$
E^{0}=E_{S O(4)}^{0}\left(\left|\eta_{z}\right|,\left|S_{z}\right|\right)+2 \mu \eta_{z}+2 \mu_{0} H S_{z}
$$

where $E_{S O(4)}^{0}(\eta, S)$ is the corresponding eigenenergy relative to the $S O(4)$-Hamiltonian (2). Note that $E_{S O(4)}^{0}(\eta, S)$ is nothing but the term $E_{S O(4)}^{0}$ of the ground-state energy (B11). Its $\eta$ and $S$ dependence can be obtained from that expression by replacing the density and spin-density dependences by $\eta$ and $S$ dependences, respectively. The energy $E_{S O(4)}^{0}(\eta, S)$ reads

$$
E_{S O(4)}^{0}(\eta, S)=\frac{N_{a}}{2 \pi} \int_{-\pi}^{\pi} d k 2 \pi \rho_{c\left(l, l^{\prime}\right)}^{0}(k)\left[\Theta\left(Q_{l, l^{\prime}}-|k|\right)\{-2 t \cos k-U / 2\}+U / 4\right]
$$

where the function $\rho_{c\left(l, l^{\prime}\right)}^{0}(k)$ is associated with the function $\rho_{s\left(l, l^{\prime}\right)}^{0}(v)$ through the integral equations (A33) and (A34) for the particular case of the ground-state distributions (B13) with $Q_{l, l^{\prime}}$ defined in Eq. (A22). These two coupled integral equations have a unique solution which defines the functions $\rho_{c\left(l, l^{\prime}\right)}^{0}(k)$ and $\rho_{s\left(l, l^{\prime}\right)}^{0}(v)$. For fixed $U$ the dependence of the energy (47) and functions $\rho_{c\left(l, l^{\prime}\right)}^{0}(k)$ and $\rho_{s\left(l, l^{\prime}\right)}^{0}(v)$ on the $\eta$ and $S$ values is defined by the following normalization equations

$$
\frac{N_{a}}{4 \pi} \int_{-\pi}^{\pi} d k 2 \pi \rho_{c\left(l, l^{\prime}\right)}^{0}(k)\left[1-\Theta\left(Q_{l, l^{\prime}}-|k|\right)\right]=\eta,
$$

and 


$$
\frac{N_{a}}{4 \pi} \int_{-\infty}^{\infty} d v 2 \pi \rho_{s\left(l, l^{\prime}\right)}^{0}(v)\left[1-\Theta\left(B_{l, l^{\prime}} / u-|v|\right)\right]=S
$$

These equations also define the dependence on $\eta$ and $S$ of the parameters $Q_{l, l^{\prime}}$ and $B_{l, l^{\prime}}$ of Eq. (A22). The use of Eqs. (13) and (14) reveals that (48) and (49) are equivalent to the normalization conditions (A36) and (A38) for the particular case of the ground-state distributions (B13). The above integral equations fully define the energy $E_{S O(4)}^{0}(\eta, S)$ and can be solved numerically. (A closed-form analytical solution is available for $E_{S O(4)}^{0}(0,0)$ [3].)

On the other hand, the energy $E(\eta, S)$ of a non-LWS and non-HWS, $\left|\eta, S ; \eta_{z}, S_{z}\right\rangle$, with the same values of $\eta_{z}$ and $S_{z}$ (but $\eta_{z} \neq \pm \eta$ and (or) $S_{z} \neq \pm S$ ) is, following Eq. (C2), given by

$$
E(\eta, S)=E_{S O(4)}^{0}(\eta, S)+2 \mu \eta_{z}+2 \mu_{0} H S_{z}
$$

where the energy $E_{S O(4)}^{0}(\eta, S)$, Eq. (47), refers to the corresponding starting states $|\eta, S ; \pm \eta, \pm S\rangle$. Following our choice, these states are also ground states of form (18), but such that $\eta \neq\left|\eta_{z}\right|$ and (or ) $S \neq\left|S_{z}\right|$.

We want to show that the energy gap

$$
E(\eta, S)-E^{0}=E_{S O(4)}^{0}(\eta, S)-E_{S O(4)}^{0}\left(\left|\eta_{z}\right|,\left|S_{z}\right|\right)
$$

where $E^{0}, E(\eta, S)$, and $E_{S O(4)}^{0}(\eta, S)$ (and $\left.E_{S O(4)}^{0}\left(\left|\eta_{z}\right|,\left|S_{z}\right|\right)\right)$ are given by Eqs. (46), (50), and (47), respectively, is positive for $\eta_{z} \neq \pm \eta$ and (or) $S_{z} \neq \pm S$. The energy $E_{S O(4)}^{0}(\eta, S)$, Eq. (47), is a monotonous increasing function of both $\eta$ and $S$, its minimum value being $E_{S O(4)}^{0}(0,0)$. This refers to the "absolute" $S O(4)$ ground state [3, 20]. Since $\eta>\left|\eta_{z}\right|$ and (or) $S>\left|S_{z}\right|\left[\right.$ if $S=\left|S_{z}\right|$ (or $\left.\eta=\left|\eta_{z}\right|\right)$ we have that $\eta>\left|\eta_{z}\right|\left(\right.$ or $\left.S>\left|S_{z}\right|\right)$, it follows that the gap (51) is always positive. Its smallest values correspond to the choices (a) $\eta=\left|\eta_{z}\right|+1$ and $S=\left|S_{z}\right|$; and (b) $\eta=\left|\eta_{z}\right|$ and $S=\left|S_{z}\right|+1$. Let us evaluate the corresponding gaps

$$
\Delta_{a}=E_{S O(4)}^{0}\left(\left|\eta_{z}\right|+1,\left|S_{z}\right|\right)-E_{S O(4)}^{0}\left(\left|\eta_{z}\right|,\left|S_{z}\right|\right)
$$

and 


$$
\Delta_{b}=E_{S O(4)}^{0}\left(\left|\eta_{z}\right|,\left|S_{z}\right|+1\right)-E_{S O(4)}^{0}\left(\left|\eta_{z}\right|,\left|S_{z}\right|\right),
$$

respectively. The gaps (52) and (53) give the excitation energy of the states ||$\eta_{z} \mid+$ $\left.1,\left|S_{z}\right| ; \eta_{z}, S_{z}\right\rangle$ and $\| \eta_{z}|,| S_{z}\left|+1 ; \eta_{z}, S_{z}\right\rangle$, respectively, relative to the LWS's and (or) HWS's, ||$\eta_{z}|,| S_{z}\left|; \eta_{z}, S_{z}\right\rangle$, of the form (18). To evaluate these gaps we use the fact that in the thermodynamic limit the excitation energy of the LWS's and (or) HWS's ||$\eta_{z}|+1,| S_{z} \mid ; \eta_{z}+$ $\left.\operatorname{sgn}\left(\eta_{z}\right) 1, S_{z}\right\rangle$ and ||$\eta_{z}|,| S_{z}\left|+1 ; \eta_{z}, S_{z}+\operatorname{sgn}\left(S_{z}\right) 1\right\rangle$ [also of form (18)] relative to the LWS and (or) HWS $\| \eta_{z}|,| S_{z}\left|; \eta_{z}, S_{z}\right\rangle$ is zero. This excitation is the spin-flip ground-state - groundstate transition (i)-(iv) studied in Ref. [20]. Its excitation energy is of order $1 / N_{a}$ and vanishes in the present thermodynamic limit. (This is a condition for the continuous character of the magnetization curve defined by Eq. (42).) This implies that

$$
E_{S O(4)}^{0}\left(\left|\eta_{z}\right|,\left|S_{z}\right|\right)+2 \mu \eta_{z}+2 \mu_{0} H S_{z}=E_{S O(4)}^{0}\left(\left|\eta_{z}\right|+1,\left|S_{z}\right|\right)+2 \mu\left[\eta_{z}+\operatorname{sgn}\left(\eta_{z}\right) 1\right]+2 \mu_{0} H S_{z},
$$

and

$$
E_{S O(4)}^{0}\left(\left|\eta_{z}\right|,\left|S_{z}\right|\right)+2 \mu \eta_{z}+2 \mu_{0} H S_{z}=E_{S O(4)}^{0}\left(\left|\eta_{z}\right|,\left|S_{z}\right|+1\right)+2 \mu \eta_{z}+2 \mu_{0} H\left[S_{z}+\operatorname{sgn}\left(S_{z}\right) 1\right] .
$$

Taking into account the relation between the signs of $\mu$ (and $H$ ) and of $\eta_{z}$ (and $S_{z}$ ) [see Eq. (29)], and combining Eqs. (52) and (53) with Eqs. (54) and (55), we finally arrive to:

$$
\Delta_{a}=2|\mu| ; \quad \Delta_{b}=2 \mu_{0}|H|
$$

These are the smallest gaps of non-LWS's and non-HWS's belonging the same canonical ensemble (i.e. having the same eigenvalues $\eta_{z}$ and $S_{z}$ ) relative to the corresponding ground state (18). Note that both (or one) of these gaps vanish(es) in the $S O(4)$ sector [or $S U(2) \otimes$ $U(1)$ and $U(1) \otimes S U(2)$ sectors] of parameter space. On the other hand, all Hamiltonian eigenstates with $\eta=0$ and (or ) $S=0$ are both LWS's and HWS's of the corresponding algebras. This implies that in the canonical ensembles with $\eta=0$ and (or ) $S=0$ there are no non-LWS's and non-HWS's singlets of the eta-spin and (or ) spin algebras. 


\section{CONCLUDING REMARKS}

In this paper we have used the pseudoparticle-operator basis and perturbation theory introduced in Refs. [11, 13, 14 to derive and study the ground states associated with all canonical ensembles belonging to the four $U(1) \otimes U(1)$ sectors of parameter space of the Hubbard chain in the presence of a magnetic field and chemical potential. Our results confirm the important role played by the pseudoparticle algebra in the low-energy physics of integrable quantum liquids: following the present study we find in Ref. [20] that the usual half-filling and zero-magnetic-field holons, antiholons, and spinons [21] correspond to a limiting case of the general pseudoparticle representation.

The simple form obtained for the ground-state, expression (18), has a deep physical meaning. It confirms [11,13] and generalizes the fact that in the pseudoparticle basis the ground state of the many-electron quantum problem is a "non-interacting" pseudoparticle ground state of simple Slater-determinant form. This also holds true for canonical ensembles belonging to sectors of higher symmetry [20,28] and, therefore, in the pseudoparticle basis the ground state of canonical ensembles of all symmetries are always states I of that simple form.

We have evaluated the energy gaps relative to the ground state of the non-LWS's and non-HWS's with common $\eta_{z}$ and $S_{z}$ eigenvalues. A complete proof of our ground-state expressions requires the calculation of the energy gaps of the states II with the same $\eta_{z}$ and $S_{z}$ eigenvalues [28].

A more general Landau-liquid theory for the sectors $U(1) \otimes U(1)$ including the states II can be constructed. These states can also be described in terms of pseudoparticles. However, in the sectors of lowest symmetry these requires, in addition to the pseudoparticles studied in this paper, new branches of "heavy" pseudoparticles 28].

The eight branches of $\alpha\left(l, l^{\prime}\right)$ pseudoparticles introduced in this paper have a deep physical meaning. This is shown in Ref. [20] where we relate the symmetry transformations of the set of pseudoparticles used in each canonical ensemble to construct the corresponding ground 
state to the symmetry of that sector. In that reference we find that the pseudoparticles of this set always transform in the representation of the corresponding group of symmetry.

Although the pseudoparticles associated with the states I are the transport carriers at

low energy [10 and couple to external potentials [12], they refer to purely non-dissipative excitations, i.e. the Hamiltonian commutes with the currents in the subspace spanned by the states I [10]. Therefore, these pseudoparticle currents give rise only to the coherent part of the conductivity spectra, i.e. to the Drude peaks 10,11,13. The finite-frequency part is associated with transitions involving the "heavy" pseudoparticles which are also needed, in the sectors of symmetry $U(1) \otimes U(1)$, to describe the states II 28 . (In the Hilbert subspace spanned by those excitations, the Hamiltonian does not commute with the current operators.)

As in the case of Landau's Fermi liquid theory [30,31, the pseudoparticle perturbation theory uses as reference state the exact ground state of the quantum problem [11,13. Also in the construction of the above generalized Landau-liquid theory referring to the Hilbert space spanned by both the states I and states II [28], the ground state which we have investigated and studied in the present paper plays a crucial role.

\section{ACKNOWLEDGMENTS}

This work was supported principally by the C.S.I.C. [Spain] and the Faculty of Sciences of the University of Lisbon. We thank D. K. Campbell, F. Guinea, and A. H. Castro Neto for stimulating discussions. N. M. R. P. is grateful for the support of A. A. Barroso and Faculty of Sciences of the University of Lisbon and for the hospitality of the C.S.I.C. [Madrid]. 


\section{APPENDIX A: BETHE-ANSATZ SOLUTION OF THE FOUR $U(1) \otimes U(1)$ SECTORS}

The BA solution associated with the Hamiltonian eigenstates I of the four $\left(l, l^{\prime}\right)$ sectors is very similar to the solution of the $(-1,-1)$ sector studied in Refs. [3, 8, 13].

In this Appendix we present the BA equations for the states I of the $\left(l, l^{\prime}\right)$ sectors of symmetry $U(1) \otimes U(1)$ and introduce the two-pseudoparticle phase shifts [10,13, 18, and other quantities needed in the expressions presented in this paper.

For each choice of $N_{c\left(l, l^{\prime}\right)}$ and $N_{s\left(l, l^{\prime}\right)}$ occupied pseudomomenta values $q_{j}$, Eq. (12), of the $c\left(l, l^{\prime}\right)$ and $s\left(l, l^{\prime}\right)$ pseudoparticles, respectively, (see the expressions of these numbers in terms of electronic numbers in Table 1) which describes one state I, Eq. (15), there is a set

of $N_{c\left(l, l^{\prime}\right)}$ real rapidity values, $k_{j}^{l, l^{\prime}}$, and other $N_{s\left(l, l^{\prime}\right)}$ real rapidity values, $v_{j}^{l, l^{\prime}}$, which are the solution of the following $N_{c\left(l, l^{\prime}\right)}+N_{s\left(l, l^{\prime}\right)}$ algebraic equations

$$
k_{j}^{l, l^{\prime}}=q_{j}+\frac{2}{N_{a}} \sum_{j^{\prime}=1}^{N_{s\left(l, l^{\prime}\right)}} \tan ^{-1}\left(v_{j^{\prime}}^{l, l^{\prime}}-(1 / u) \sin k_{j}^{l, l^{\prime}}\right), \quad j=1, \ldots, N_{c\left(l, l^{\prime}\right)},
$$

and

$$
\begin{aligned}
q_{j} & =\frac{2}{N_{a}} \sum_{j^{\prime}=1}^{N_{c\left(l, l^{\prime}\right)}} \tan ^{-1}\left(v_{j}^{l, l^{\prime}}-(1 / u) \sin k_{j^{\prime}}^{l, l^{\prime}}\right) \\
& -\frac{2}{N_{a}} \sum_{j^{\prime}=1}^{N_{s\left(l, l^{\prime}\right)}} \tan ^{-1}\left(\frac{1}{2}\left(v_{j}^{l, l^{\prime}}-v_{j^{\prime}}^{l, l^{\prime}}\right)\right), \quad j=1, \ldots, N_{s\left(l, l^{\prime}\right)} .
\end{aligned}
$$

In the thermodynamic limit $\left(N_{\alpha\left(l, l^{\prime}\right)}, N_{a} \rightarrow \infty\right.$ with $n_{\alpha\left(l, l^{\prime}\right)}=N_{\alpha\left(l, l^{\prime}\right)} / N_{a}$ finite $)$ the rapidity values $k_{j}^{l, l^{\prime}}$ and $v_{j}^{l, l^{\prime}}$ give rise to rapidity functions $K_{l, l^{\prime}}(q)$ and $S_{l, l^{\prime}}\left(q^{\prime}\right)$, respectively, which are eigenvalues of the corresponding rapidity operators [13]. The set of algebraic equations (A1)-(A2) lead to the following two coupled integral equations

$$
K_{l, l^{\prime}}(q)=q+\frac{1}{\pi} \int_{q_{s\left(l, l^{\prime}\right)}^{(-)}}^{q_{s\left(l, l^{\prime}\right)}^{(+)}} d q^{\prime} N_{s\left(l, l^{\prime}\right)}\left(q^{\prime}\right) \tan ^{-1}\left(S_{l, l^{\prime}}\left(q^{\prime}\right)-(1 / u) \sin K_{l, l^{\prime}}(q)\right),
$$

and

$$
\begin{aligned}
q & =\frac{1}{\pi} \int_{q_{c\left(l, l^{\prime}\right)}^{(-)}}^{q_{c\left(l, l^{\prime}\right)}^{(+)}} d q^{\prime} N_{c\left(l, l^{\prime}\right)}\left(q^{\prime}\right) \tan ^{-1}\left(S_{l, l^{\prime}}(q)-(1 / u) \sin K_{l, l^{\prime}}\left(q^{\prime}\right)\right) \\
& -\frac{1}{\pi} \int_{q_{s\left(l, l^{\prime}\right)}^{(-)}}^{q_{s\left(l, l^{\prime}\right)}^{(+)}} d q^{\prime} N_{s\left(l, l^{\prime}\right)}\left(q^{\prime}\right) \tan ^{-1}\left(\frac{1}{2}\left(S_{l, l^{\prime}}(q)-S_{l, l^{\prime}}\left(q^{\prime}\right)\right)\right)
\end{aligned}
$$


respectively, where the limits of the pseudo-Brillouin zones, $q_{\alpha\left(l, l^{\prime}\right)}^{( \pm)}$, are given by Eqs. (19) (21) with $N_{\alpha\left(l, l^{\prime}\right)}$ replaced by $N_{\alpha\left(l, l^{\prime}\right)}^{*}$ and the pseudomomentum distributions, $N_{c\left(l, l^{\prime}\right)}(q)$, are the eigenvalues (and also expectation values) of the operators (26) relatively to the states I of form (15), ie

$$
N_{\alpha\left(l, l^{\prime}\right)}(q)=\left\langle\eta_{z}, S_{z}\left|\hat{N}_{\alpha\left(l, l^{\prime}\right)}(q)\right| \eta_{z}, S_{z}\right\rangle
$$

The pseudomomentum distribution $N_{\alpha\left(l, l^{\prime}\right)}(q)(\mathrm{A} 5)$ is 1 and 0 for occupied and nonoccupied pseudomomenta, respectively, of the states (15). Therefore, the distributions (A5) fully define these Hamiltonian eigenstates I [see Eq. (27)]. We have that

$$
\frac{N_{a}}{2 \pi} \int_{q_{\alpha\left(l, l^{\prime}\right)}^{(-)}}^{q_{\alpha\left(l, l^{\prime}\right)}^{(+)}} d q=N_{\alpha\left(l, l^{\prime}\right)}^{*}, \quad \frac{N_{a}}{2 \pi} \int_{q_{\alpha\left(l, l^{\prime}\right)}^{(-)}}^{q_{\alpha\left(l, l^{\prime}\right)}^{(+)}} d q N_{\alpha\left(l, l^{\prime}\right)}(q)=N_{\alpha\left(l, l^{\prime}\right)},
$$

where $N_{\alpha\left(l, l^{\prime}\right)}^{*}$ and $N_{\alpha\left(l, l^{\prime}\right)}$ are the number of available $\alpha\left(l, l^{\prime}\right)$ pseudomomentum values , $q_{j}$ [see Eq. (12)], and numbers of $\alpha\left(l, l^{\prime}\right)$ pseudoparticles, respectively, given in Table 1.

For each Hamiltonian eigenstate (15) there is one, and only one, pair of rapidity eigenvalues $K_{l, l^{\prime}}(q)$ and $S_{l, l^{\prime}}(q)$. These are functionals of the pseudomomentum distributions. The solution of Eqs. (A3) and (A4) provides these rapidity functionals of the pseudomomentum distributions $N_{\alpha\left(l, l^{\prime}\right)}(q)$.

It is easier to express the rapidity functions in terms of the eigenvalues of the normalordered operators (30) and (43), which define the pseudomomentum deviations. The rapidity functions can then be expanded in these deviations as [13, 18

$$
K_{l, l^{\prime}}(q)=K_{l, l^{\prime}}^{(0)}(q)+K_{l, l^{\prime}}^{(1)}(q)+K_{l, l^{\prime}}^{(2)}(q)+\ldots
$$

and

$$
S_{l, l^{\prime}}(q)=S_{l, l^{\prime}}^{(0)}(q)+S_{l, l^{\prime}}^{(1)}(q)+S_{l, l^{\prime}}^{(2)}(q)+\ldots
$$

where $K_{l, l^{\prime}}^{(j)}(q)$ and $S_{l, l^{\prime}}^{(j)}(p)$ are the $j$ th-order terms. Equations (A3) and (A4) allow the systematic evaluation order by order of all terms of the expansions (A7) and (A8). As shown in Ref. 13 for the case of the $(-1,-1)$ sector, this deviation expansion corresponds to a 
operator expansion in the pseudoparticle scattering order. The possibility of such expansion follows from the perturbative character of the pseudoparticle operator basis [11, 13, 18].

Here we are interessed in the first-order terms of (A7) and (A8) which involve the twopseudoparticle phase shifts [10,13, 18]. By using a recursion procedure, we find that the rapidities (A7) and (A8) may be simply written as

$$
K_{l, l^{\prime}}(q)=K_{l, l^{\prime}}^{(0)}\left(\mathcal{Q}_{l, l^{\prime}}(q)\right), \quad S_{l, l^{\prime}}(q)=S_{l, l^{\prime}}^{(0)}\left(\mathcal{P}_{l, l^{\prime}}(q)\right)
$$

where $K_{l, l^{\prime}}^{(0)}(q)$ and $S_{l, l^{\prime}}^{(0)}(q)$ are the solutions that correspond to the choice of distribution (B3) of Appendix B (in this paper we want to confirm that this choice defines the ground state) and $\mathcal{Q}_{l, l^{\prime}}(q)$ and $\mathcal{P}_{l, l^{\prime}}(q)$ are functionals of the form

$$
\begin{aligned}
& \mathcal{Q}_{l, l^{\prime}}(q)=q+\mathcal{Q}_{l, l^{\prime}}^{(1)}(q)+\mathcal{Q}_{l, l^{\prime}}^{(2)}(q)+\ldots \\
& \mathcal{P}_{l, l^{\prime}}(q)=q+\mathcal{P}_{l, l^{\prime}}^{(1)}(q)+\mathcal{P}_{l, l^{\prime}}^{(2)}(q)+\ldots
\end{aligned}
$$

which can be obtained by solving Eqs. (A3) and (A4) order by order. The results (A9)(A11) imply that the first-order terms of the rhs of Eqs. (A7) and (A8) may be written as

$$
K_{l, l^{\prime}}^{(1)}(q)=\frac{d K_{l, l^{\prime}}^{(0)}(q)}{d q} \mathcal{Q}_{l, l^{\prime}}^{(1)}(q)
$$

and

$$
S_{l, l^{\prime}}^{(1)}(q)=\frac{d S_{l, l^{\prime}}^{(0)}(q)}{d q} \mathcal{P}_{l, l^{\prime}}^{(1)}(q)
$$

respectively. We note that the functions $d K_{l, l^{\prime}}^{(0)}(q) / d q$ and $d S_{l, l^{\prime}}^{(0)}(q) / d q$ obey the equations

$$
\frac{d K_{l, l^{\prime}}^{(0)}(q)}{d q}=\frac{1}{2 \pi \rho_{c\left(l, l^{\prime}\right)}^{0}\left(K_{l, l^{\prime}}^{(0)}(q)\right)}
$$

and

$$
\frac{d S_{l, l^{\prime}}^{(0)}(q)}{d q}=\frac{1}{2 \pi \rho_{s\left(l, l^{\prime}\right)}^{0}\left(S_{l, l^{\prime}}^{(0)}(q)\right)}
$$


respectively, where the functions $2 \pi \rho_{c\left(l, l^{\prime}\right)}^{0}(k)$ and $2 \pi \rho_{s\left(l, l^{\prime}\right)}^{0}(v)$ are the "ground-state" solutions of Eqs. (A33) and (A34) below. [The functions $2 \pi \rho_{c(-1,-1)}^{0}(k)$ and $2 \pi \rho_{s(-1,-1)}^{0}(v)$ (with $\rho_{s(-1,-1)}^{0}(v)=u \sigma(u v)$, where $\left.v=\Lambda / u\right)$ are the usual ground-state distributions of Lieb and $\mathrm{Wu}$ [3].]

Solving Eqs. (A3) and (A4) to first order leads to

$$
\begin{aligned}
& \mathcal{Q}^{(1)}(q)=\sum_{\alpha} \int_{q_{\alpha\left(l, l^{\prime}\right)}^{(-)}}^{q_{\alpha\left(l, l^{\prime}\right)}^{(+)}} d q^{\prime} \delta N_{l, l^{\prime}}\left(q^{\prime}\right) \Phi_{c \alpha}^{l, l^{\prime}}\left(q, q^{\prime}\right), \\
& \mathcal{P}^{(1)}(q)=\sum_{\alpha} \int_{q_{\alpha\left(l, l^{\prime}\right)}^{(-)}}^{q_{\alpha\left(l, l^{\prime}\right)}^{(+)}} d q^{\prime} \delta N_{l, l^{\prime}}\left(q^{\prime}\right) \Phi_{s \alpha}^{l, l^{\prime}}\left(q, q^{\prime}\right),
\end{aligned}
$$

where $\delta N_{\alpha\left(l, l^{\prime}\right)}(q)=\left\langle\eta_{z}, S_{z}\left|: \hat{N}_{\alpha\left(l, l^{\prime}\right)}(q):\right| \eta_{z}, S_{z}\right\rangle$. The four two-pseudoparticle phase shifts $\Phi_{\alpha \alpha^{\prime}}^{l, l^{\prime}}\left(q, q^{\prime}\right)$ can be written as

$$
\begin{gathered}
\Phi_{c c}^{l, l^{\prime}}\left(q, q^{\prime}\right)=\bar{\Phi}_{c c}^{l, l^{\prime}}\left(\frac{\sin K_{l, l^{\prime}}^{(0)}(q)}{u}, \frac{\sin K_{l, l^{\prime}}^{(0)}\left(q^{\prime}\right)}{u}\right), \\
\Phi_{c s}^{l, l^{\prime}}\left(q, q^{\prime}\right)=\bar{\Phi}_{c s}^{l, l^{\prime}}\left(\frac{\sin K_{l, l^{\prime}}^{(0)}(q)}{u}, S_{l, l^{\prime}}^{(0)}\left(q^{\prime}\right)\right) \\
\Phi_{s c}^{l, l^{\prime}}\left(q, q^{\prime}\right)=\bar{\Phi}_{s c}^{l, l^{\prime}}\left(S_{l, l^{\prime}}^{(0)}(q), \frac{\sin K_{l, l^{\prime}}^{(0)}\left(q^{\prime}\right)}{u}\right) \\
\Phi_{s s}^{l, l^{\prime}}\left(q, q^{\prime}\right)=\bar{\Phi}_{s s}^{l, l^{\prime}}\left(S_{l, l^{\prime}}^{(0)}(q), S_{l, l^{\prime}}^{(0)}\left(q^{\prime}\right)\right) .
\end{gathered}
$$

Introducing the parameters

$$
Q_{l, l^{\prime}}=K_{l, l^{\prime}}^{(0)}\left(q_{F c\left(l, l^{\prime}\right)}\right), \quad B_{l, l^{\prime}} / u=S_{l, l^{\prime}}^{(0)}\left(q_{F s\left(l, l^{\prime}\right)}\right)
$$

and

$$
x_{l, l^{\prime}}^{0}=\frac{\sin Q_{l, l^{\prime}}}{u}, \quad y_{l, l^{\prime}}^{0}=B_{l, l^{\prime}} / u
$$

$\left(Q_{-1,-1}\right.$ and $B_{-1,-1}$ are the usual cutoff parameters of the ground-state Lieb-Wu equations [3]) we find that the phase shifts $\bar{\Phi}_{\alpha \alpha^{\prime}}^{l, l^{\prime}}$ obey the following integral equations: 


$$
\begin{gathered}
\bar{\Phi}_{c c}^{l, l^{\prime}}\left(x, x^{\prime}\right)=\frac{1}{\pi} \int_{-y_{l, l^{\prime}}^{0}}^{y_{l, l^{\prime}}^{0}} d y^{\prime \prime} \frac{\bar{\Phi}_{s c}^{l, l^{\prime}}\left(y^{\prime \prime}, x^{\prime}\right)}{1+\left(x-y^{\prime \prime}\right)^{2}}, \\
\bar{\Phi}_{c s}^{l, l^{\prime}}\left(x, y^{\prime}\right)=-\frac{1}{\pi} \tan ^{-1}\left(x-y^{\prime}\right)+\frac{1}{\pi} \int_{-y_{l, l^{\prime}}^{0}}^{y_{l, l^{\prime}}^{0}} d y^{\prime \prime} \frac{\bar{\Phi}_{s s}^{l, l^{\prime}}\left(y^{\prime \prime}, y^{\prime}\right)}{1+\left(x-y^{\prime \prime}\right)^{2}}, \\
\bar{\Phi}_{s c}^{l, l^{\prime}}\left(y, x^{\prime}\right)=-\frac{1}{\pi} \tan ^{-1}\left(y-x^{\prime}\right)+\int_{-y_{l, l^{\prime}}^{0}}^{y_{l, l^{\prime}}^{0}} d y^{\prime \prime} G\left(y, y^{\prime \prime}\right) \bar{\Phi}_{s c}^{l, l^{\prime}}\left(y^{\prime \prime}, x^{\prime}\right), \\
\bar{\Phi}_{s s}^{l, l^{\prime}}\left(y, y^{\prime}\right)=\frac{1}{\pi} \tan ^{-1}\left(\frac{y-y^{\prime}}{2}\right)-\frac{1}{\pi^{2}} \int_{-x_{l, l^{\prime}}^{0}}^{x_{l, l^{\prime}}^{0}} d x^{\prime \prime} \frac{\tan ^{-1}\left(x^{\prime \prime}-y^{\prime}\right)}{1+\left(y-x^{\prime \prime}\right)^{2}} \\
+\int_{-y_{l, l^{\prime}}^{0}}^{y_{l, l^{\prime}}^{0}} d y^{\prime \prime} G\left(y, y^{\prime \prime}\right) \bar{\Phi}_{s s}^{l, l^{\prime}}\left(y^{\prime \prime}, y^{\prime}\right) .
\end{gathered}
$$

The kernel $G\left(y, y^{\prime}\right)$ reads 18

$$
G\left(y, y^{\prime}\right)=-\frac{1}{2 \pi}\left[\frac{1}{1+\left(\left(y-y^{\prime}\right) / 2\right)^{2}}\right]\left[1-\frac{1}{2}\left(t(y)+t\left(y^{\prime}\right)+\frac{l(y)-l\left(y^{\prime}\right)}{y-y^{\prime}}\right)\right]
$$

where

$$
t(y)=\frac{1}{\pi} \sum_{j= \pm 1}(j) \tan ^{-1}\left(y+j x_{l, l^{\prime}}^{0}\right)
$$

and

$$
l(y)=\frac{1}{\pi} \sum_{j= \pm 1}(j) \ln \left(1+\left(y+j x_{l, l^{\prime}}^{0}\right)^{2}\right) .
$$

It is useful to introduce an alternative representation for the rapidity functions $K_{l, l^{\prime}}(q)$ and $S_{l, l^{\prime}}(q)$ of Eqs. (A3) and (A4) in terms of distributions $\rho_{c\left(l, l^{\prime}\right)}(k)$ and $\rho_{s\left(l, l^{\prime}\right)}(v)$. This leads to new equations which are equivalent to the latter equations. This second representation is less appropriate for the pseudoparticle operator basis but was, historically, the most widely used in BA problems [3,8, 16]. The reason is that it leads to integral equations which, in some limits, are of mathematical standard type. However, and as we discuss below, the representation associated with the Eqs. (A3) and (A4) has a clearer physical connection to the BA operator algebra.

Let us introduce the function $\rho_{c\left(l, l^{\prime}\right)}(k)$ such that 


$$
2 \pi \rho_{c\left(l, l^{\prime}\right)}\left(K_{l, l^{\prime}}(q)\right)=1 /\left[\frac{d K_{l, l^{\prime}}(q)}{d q}\right]
$$

This function is related to the distribution $\rho_{s\left(l, l^{\prime}\right)}(v)$ which is defined as

$$
2 \pi \rho_{s\left(l, l^{\prime}\right)}\left(S_{l, l^{\prime}}(q)\right)=1 /\left[\frac{d S_{l, l^{\prime}}(q)}{d q}\right]
$$

Equations (A31) and (A32) define the transformation $q \rightarrow k$ for $\alpha\left(l, l^{\prime}\right)=c\left(l, l^{\prime}\right)$ and $q \rightarrow v$ for $\alpha\left(l, l^{\prime}\right)=s\left(l, l^{\prime}\right)$, where the new variable $k$ varies between $K\left(q_{c\left(l, l^{\prime}\right)}^{(-)}\right)$and $K\left(q_{c\left(l, l^{\prime}\right)}^{(+)}\right)$and the variable $v$ runs from $S\left(q_{s\left(l, l^{\prime}\right)}^{(-)}\right)$to $S\left(q_{s\left(l, l^{\prime}\right)}^{(+)}\right)$.

Combining Eqs. (A3),(A4) and (A31),(A32) we find that $\rho_{c\left(l, l^{\prime}\right)}(k)$ and $\rho_{s\left(l, l^{\prime}\right)}(v)$ are the solutions of the following system of coupled integral equations

$$
2 \pi \rho_{c\left(l, l^{\prime}\right)}(k)=1+\frac{1}{\pi} \frac{\cos k}{u} \int_{S\left(q_{s\left(l, l^{\prime}\right)}^{(-)}\right)}^{S\left(q_{s\left(l, l^{\prime}\right)}^{(+)}\right)} d v^{\prime} \tilde{N}_{s\left(l, l^{\prime}\right)}\left(v^{\prime}\right) \frac{2 \pi \rho_{s\left(l, l^{\prime}\right)}\left(v^{\prime}\right)}{1+\left[v^{\prime}-\frac{\sin k}{u}\right]^{2}}
$$

and

$$
\begin{aligned}
2 \pi \rho_{s\left(l, l^{\prime}\right)}(v) & =\frac{1}{\pi} \int_{K_{l, l^{\prime}}\left(q_{c\left(l, l^{\prime}\right)}^{(-)}\right)}^{K\left(q_{c\left(l, l^{\prime}\right)}^{(+)}\right)} d k^{\prime} \tilde{N}_{c\left(l, l^{\prime}\right)}\left(k^{\prime}\right) \frac{2 \pi \rho_{s\left(l, l^{\prime}\right)}\left(k^{\prime}\right)}{1+\left[v-\frac{\sin k^{\prime}}{u}\right]^{2}} \\
& -\frac{1}{2 \pi} \int_{S_{l, l^{\prime}}\left(q_{s\left(l, l^{\prime}\right)}^{(-)}\right)}^{S\left(q_{s\left(l, l^{\prime}\right.}^{(+)}\right)} d v^{\prime} \tilde{N}_{s\left(l, l^{\prime}\right)}\left(v^{\prime}\right) \frac{2 \pi \rho_{s\left(l, l^{\prime}\right)}\left(v^{\prime}\right)}{1+\left[\frac{1}{2}\left(v-v^{\prime}\right)\right]^{2}}
\end{aligned}
$$

where $\tilde{N}_{c\left(l, l^{\prime}\right)}(k)$ and $\tilde{N}_{s\left(l, l^{\prime}\right)}(v)$ are the representation of the distributions $N_{c\left(l, l^{\prime}\right)}(q)$ and $N_{s\left(l, l^{\prime}\right)}(q)$ (A5), respectively, in the $k, v$ space associated with the transformation $q \rightarrow k$ $\left[\right.$ for $\left.\alpha=c\left(l, l^{\prime}\right)\right]$ and $q \rightarrow v\left[\right.$ for $\left.\alpha=s\left(l, l^{\prime}\right)\right]$.

For $\left(l, l^{\prime}\right)=(-1,-1)$ Eqs. (A33) and (A34) are similar to Eqs. (A4) and (A5), respectively, of Ref. [17]. However, Eqs. (A33) and (A34) are more general: the limits of integration of Eqs. (A4) and (A5) of Ref. [17] are only valid for Hamiltonian eigenstates differing by a small density of excited pseudoparticles from (18) (they are also valid for all eigenstates (15) where the occupied pseudomomenta of the functions (A5) are distributed symmetrically around the origin), whereas Eqs. (A33) and (A34) are valid in each canonical ensemble for all states I (15) in number of (17).

For the particular case of a $(-1,-1)$ state (18) the functions (A31) and (A32) are nothing but the usual distributions of Lieb and $\mathrm{Wu}$ [3, 17]. Equations (A33) and (A34) give the generalization for all states I (15). 
Following Eqs. (A6), (A31), and (A32) the distributions $\rho_{c\left(l, l^{\prime}\right)}(k)$ and $\rho_{s\left(l, l^{\prime}\right)}(v)$ obey the normaliztion conditions

$$
\begin{gathered}
\frac{N_{a}}{2 \pi} \int_{K_{l, l^{\prime}}\left(q_{c\left(l, l^{\prime}\right)}^{(-)}\right)}^{K_{l, l^{\prime}}\left(q_{c\left(l, l^{\prime}\right)}^{(+)}\right)} d k 2 \pi \rho_{c\left(l, l^{\prime}\right)}(k)=N_{c\left(l, l^{\prime}\right)}^{*}, \\
\frac{N_{a}}{2 \pi} \int_{K_{l, l^{\prime}}\left(q_{c\left(l, l^{\prime}\right)}^{(-)}\right)}^{K_{l, l^{\prime}}\left(q_{c\left(l, l^{\prime}\right)}^{(+)}\right)} d k 2 \pi \rho_{c\left(l, l^{\prime}\right)}(k) \tilde{N}_{c\left(l, l^{\prime}\right)}(k)=N_{c\left(l, l^{\prime}\right),} \\
\frac{N_{a}}{2 \pi} \int_{S_{l, l^{\prime}}\left(q_{s\left(l, l^{\prime}\right)}^{(-)}\right)}^{S_{l, l^{\prime}}^{((+)}\left(q_{s\left(l, l^{\prime}\right)}^{(+)}\right)} d k 2 \pi \rho_{s\left(l, l^{\prime}\right)}(k)=N_{s\left(l, l^{\prime}\right)}^{*}, \\
\frac{N_{a}}{2 \pi} \int_{S_{l, l^{\prime}}\left(q_{s\left(l, l^{\prime}\right)}^{(-)}\right)}^{S_{l, l^{\prime}}\left(q_{s\left(l, l^{\prime}\right)}^{(+)}\right)} d k 2 \pi \rho_{s\left(l, l^{\prime}\right)}(k) \tilde{N}_{s\left(l, l^{\prime}\right)}(k)=N_{s\left(l, l^{\prime}\right)} .
\end{gathered}
$$

[Here the numbers $N_{\alpha\left(l, l^{\prime}\right)}^{*}$ and $N_{\alpha\left(l, l^{\prime}\right)}$ of the four $\left(l, l^{\prime}\right)$ sectors of symmetry $U(1) \otimes U(1)$ are given in Table 1.]

As mentioned above, the representation associated with the distributions $\rho_{c(-1,-1)}(k)$ and $\rho_{s(-1,-1)}(v)$ was, until recently, the most used in BA in what concerns the description of states of form (18) and states whose distributions of BA quantum numbers differ from (18) by a vanishing density of these numbers [3, 8, 16]. One of the reasons for this is that from the mathematical point of view the integral equations (A33) and (A34) are, for these states, easier to handle than Eqs. (A3) and (A4). However, while the distributions (A5) of the latter equations are expectation values of the operators (26) and, therefore, have a clear physical meaning, we note that none of the distributions and functions $\rho_{c\left(l, l^{\prime}\right)}(k)$ and $\rho_{s\left(l, l^{\prime}\right)}(v), \tilde{N}_{c\left(l, l^{\prime}\right)}(k)$ and $\tilde{N}_{s\left(l, l^{\prime}\right)}(v)$, and $\rho_{c\left(l, l^{\prime}\right)}(k) \tilde{N}_{c\left(l, l^{\prime}\right)}(k)$ and $\rho_{s\left(l, l^{\prime}\right)}(v) \tilde{N}_{s\left(l, l^{\prime}\right)}(v)$ are expectation values of any operator. (See, for instance, Fig. 3 of Ref. 32 where $\rho_{c(-1,-1)}(k)$ and the electronic momentum distribution are compared for zero magnetic field.) The latter distributions and functions are just a useful mathematical representation in the $k, v$ space for the pseudomomentum distributions (A5) and eigenvalues $K_{l, l^{\prime}}(q)$ and $S_{l, l^{\prime}}(q)$ of the Hamiltonian eigenstates (15). 
Finally, the expressions of the pseudoparticle bands (34) - (36) involve a third representation for the two-pseudoparticle phase shifts (A18)-(A21) and (A24)-(A27) in terms of the $k, v$ space variables. The corresponding phase shifts are given by

$$
\begin{gathered}
\tilde{\Phi}_{c c}^{l, l^{\prime}}\left(k, k^{\prime}\right)=\bar{\Phi}_{c c}^{l, l^{\prime}}\left(\frac{\sin k}{u}, \frac{\sin k^{\prime}}{u}\right), \\
\tilde{\Phi}_{c s}^{l, l^{\prime}}\left(k, v^{\prime}\right)=\bar{\Phi}_{c s}^{l, l^{\prime}}\left(\frac{\sin k}{u}, v^{\prime}\right), \\
\tilde{\Phi}_{s c}^{l, l^{\prime}}\left(v, k^{\prime}\right)=\bar{\Phi}_{s c}^{l, l^{\prime}}\left(v, \frac{\sin k^{\prime}}{u}\right),
\end{gathered}
$$

and

$$
\tilde{\Phi}_{s s}^{l, l^{\prime}}\left(v, v^{\prime}\right)=\bar{\Phi}_{s s}^{l, l^{\prime}}\left(v, v^{\prime}\right)
$$

where the functions $\bar{\Phi}_{\alpha \alpha^{\prime}}^{l, l^{\prime}}$ are defined by Eqs. (A24)-(A27).

In Appendix B we study the energy of the Hamiltonian eigenstates I associated with the BA Eqs. (A3)-(A4) and (A33)-(A34). 


\section{APPENDIX B: GENERAL ENERGY EXPRESSION FOR THE STATES I}

In this Appendix we present and study the general energy expression for the states I with common eigenvalues $\eta_{z}$ and $S_{z}$ corresponding to the $\left(l, l^{\prime}\right)$ sectors of symmetry $U(1) \otimes U(1)$.

The Hamiltonian eigenstates (15) are also eigenstates of the rapidity operator $\hat{K}_{l, l^{\prime}}(q)$ and of the operators (26). Their energies can be written as

$$
E=E_{S O(4)}-|\mu|\left[N_{c\left(l, l^{\prime}\right)}^{*}-N_{c\left(l, l^{\prime}\right)}\right]-\mu_{0}|H|\left[N_{s\left(l, l^{\prime}\right)}^{*}-N_{s\left(l, l^{\prime}\right)}\right],
$$

where

$$
E_{S O(4)}=\frac{N_{a}}{2 \pi} \int_{q_{c\left(l, l^{\prime}\right)}^{(-)}}^{q_{c\left(l, l^{\prime}\right)}^{(+)}} d q\left[N_{c\left(l, l^{\prime}\right)}(q)\left\{-2 t \cos K_{l, l^{\prime}}(q)-U / 2\right\}+U / 4\right] .
$$

Here $N_{c\left(l, l^{\prime}\right)}(q)$ is the $c\left(l, l^{\prime}\right)$ pseudomomentum distribution (A5) and $K_{l, l^{\prime}}(q)$ is the eigenvalue of the rapidity operator $\hat{K}_{l, l^{\prime}}(q)$ defined by Eqs. (A3) and (A4): the rapidity eigenvalues of the states I of form (15), $\left|\eta_{z}, S_{z}\right\rangle$, are fully determined by the distributions (A5) through the BA equations (A3) and (A4). (In these equations $S_{l, l^{\prime}}(q)$ is the eigenvalue of the rapidity operator $\hat{S}_{l, l^{\prime}}(q)$.)

For the case of the state (18) we denote the pseudomomentum distribution (26) by $N_{\alpha\left(l, l^{\prime}\right)}^{0}(q)$. It is given by

$$
\begin{aligned}
N_{\alpha\left(l, l^{\prime}\right)}^{0}(q) & =\left\langle 0, \eta_{z}, S_{z}\left|\hat{N}_{\alpha\left(l, l^{\prime}\right)}(q)\right| 0, \eta_{z}, S_{z}\right\rangle \\
& =\Theta\left(q_{F \alpha\left(l, l^{\prime}\right)}^{(+)}-q\right), \quad 0<q<q_{\alpha\left(l, l^{\prime}\right)}^{(+)} \\
& =\Theta\left(q-q_{F \alpha\left(l, l^{\prime}\right)}^{(-)}\right), \quad q_{\alpha\left(l, l^{\prime}\right)}^{(-)}<q<0 .
\end{aligned}
$$

It is useful to express the energy (B2) in terms of the distribution $\rho_{c\left(l, l^{\prime}\right)}(k)$ of Eqs. (A33) and (A34). The term (B2) of the energy (B1) can be rewritten in terms of that function as follows

$$
E_{S O(4)}=\frac{N_{a}}{2 \pi} \int_{K_{l, l^{\prime}}\left(q_{c\left(l, l^{\prime}\right)}^{(-)}\right)}^{K_{l, l^{\prime}}\left(q_{c\left(l, l^{\prime}\right.}^{(+)}\right)} d k 2 \pi \rho_{c\left(l, l^{\prime}\right)}(k)\left[\tilde{N}_{c\left(l, l^{\prime}\right)}(k)\{-2 t \cos k-U / 2\}+U / 4\right],
$$

where $\tilde{N}_{c\left(l, l^{\prime}\right)}(k)$ is the distribution of Eqs. (A33) and (A34). The two energy expressions (B1) with $E_{S O(4)}$ given by (B2) and (B4), respectively, are equivalent. For the sake of clarity 
we have here expressed the term $E_{S O(4)}$ of the energy (B1) in both the forms (B2) and (B4). These energies can be obtained by solving numerically Eqs. (A3), (A4), and (B2) or Eqs. (A33), (A34), and (B4).

As mentioned in Sec. III, in each canonical ensemble of eigenvalues $\eta_{z}$ and $S_{z}$ the state I of maximal energy corresponds to the pseudomomentum distribution (A5) of the particular form

$$
\begin{aligned}
N_{\alpha\left(l, l^{\prime}\right)}^{*}(q) & =\left\langle *, \eta_{z}, S_{z}\left|\hat{N}_{\alpha\left(l, l^{\prime}\right)}(q)\right| *, \eta_{z}, S_{z}\right\rangle \\
& =1-\Theta\left(q_{* \alpha\left(l, l^{\prime}\right)}^{(+)}-q\right), \quad 0<q<q_{\alpha\left(l, l^{\prime}\right)}^{(+)} \\
& =1-\Theta\left(q-q_{* \alpha\left(l, l^{\prime}\right)}^{(-)}\right), \quad q_{\alpha\left(l, l^{\prime}\right)}^{(-)}<q<0,
\end{aligned}
$$

where

$$
q_{* \alpha\left(l, l^{\prime}\right)}^{( \pm)}=q_{\alpha\left(l, l^{\prime}\right)}^{( \pm)}-q_{F \alpha\left(l, l^{\prime}\right)}^{( \pm)}
$$

Except for terms of $1 / N_{a}$ order, the pseudomomenta (B6) can be written as

$$
q_{* \alpha\left(l, l^{\prime}\right)}^{( \pm)} \approx \pm q_{* \alpha\left(l, l^{\prime}\right)}
$$

where

$$
q_{* \alpha\left(l, l^{\prime}\right)}=q_{\alpha\left(l, l^{\prime}\right)}-q_{F \alpha\left(l, l^{\prime}\right)}=\frac{\pi}{N_{a}}\left[N_{\alpha\left(l, l^{\prime}\right)}^{*}-N_{\alpha\left(l, l^{\prime}\right)}\right]
$$

It follows that

$$
q_{* c\left(l, l^{\prime}\right)}=q_{c\left(l, l^{\prime}\right)}-q_{F c\left(l, l^{\prime}\right)}, \quad q_{* s\left(l, l^{\prime}\right)}=q_{s\left(l, l^{\prime}\right)}-q_{F s\left(l, l^{\prime}\right)}
$$

The state I $\left|*, \eta_{z}, S_{z}\right\rangle$ of the rhs of Eq. (B5) is of the form

$$
\left|* ; \eta_{z}, S_{z}\right\rangle=\prod_{\alpha=c, s}\left[\prod_{q=q_{\alpha\left(l, l^{\prime}\right)}^{(-)}}^{q_{* \alpha\left(l, l^{\prime}\right)}^{(-)}} \prod_{q=q_{* \alpha\left(l, l^{\prime}\right)}^{(+)}}^{q_{\alpha\left(l, l^{\prime}\right)}^{(+)}} b_{q \alpha\left(l, l^{\prime}\right)}^{\dagger}\right]\left|V ; l, l^{\prime}\right\rangle .
$$

Inserting the distributions (B3) and (B5) in Eqs. (A3) and (A4) defines the rapidities $K_{l, l^{\prime}}^{(0)}(q), S_{l, l^{\prime}}^{(0)}(q)$ and $K_{l, l^{\prime}}^{(*)}(q), S_{l, l^{\prime}}^{(*)}(q)$, respectively. (The rapidity functions $K_{l, l^{\prime}}^{(0)}(q)$ and $S_{l, l^{\prime}}^{(0)}(q)$ are also defined by Eq. (36).) We call the corresponding energies 


$$
E_{0}=E_{S O(4)}^{0}-|\mu|\left[N_{c\left(l, l^{\prime}\right)}^{*}-N_{c\left(l, l^{\prime}\right)}\right]-\mu_{0}|H|\left[N_{s\left(l, l^{\prime}\right)}^{*}-N_{s\left(l, l^{\prime}\right)}\right]
$$

and

$$
E_{*}=E_{S O(4)}^{*}-|\mu|\left[N_{c\left(l, l^{\prime}\right)}^{*}-N_{c\left(l, l^{\prime}\right)}\right]-\mu_{0}|H|\left[N_{s\left(l, l^{\prime}\right)}^{*}-N_{s\left(l, l^{\prime}\right)}\right]
$$

respectively, where $E_{S O(4)}^{0}$ and $E_{S O(4)}^{*}$ are given by (B2) with the $c\left(l, l^{\prime}\right)$ pseudomomentum distribution defined by (B3) and (B5), respectively, and the rapidities given by $K_{l, l^{\prime}}^{(0)}(q)$ and $K_{l, l^{\prime}}^{(*)}(q)$, respectively.

The energies $E_{S O(4)}^{0}$ and $E_{S O(4)}^{*}$ can also be written in the form (B4). Let us introduce the corresponding functions $\rho_{c\left(l, l^{\prime}\right)}^{0}(k), \rho_{s\left(l, l^{\prime}\right)}^{0}(v)$ and $\rho_{c\left(l, l^{\prime}\right)}^{*}(k), \rho_{s\left(l, l^{\prime}\right)}^{*}(v)$, respectively. These are solutions of the integral equations (A33) and (A34) with

$$
\tilde{N}_{c\left(l, l^{\prime}\right)}^{0}(k)=\Theta\left(Q_{l, l^{\prime}}-|k|\right), \quad \tilde{N}_{s\left(l, l^{\prime}\right)}^{0}(v)=\Theta\left(B_{l, l^{\prime}} / u-|v|\right),
$$

and

$$
\tilde{N}_{c\left(l, l^{\prime}\right)}^{*}(k)=1-\Theta\left(Q_{l, l^{\prime}}^{*}-|k|\right), \quad \tilde{N}_{s\left(l, l^{\prime}\right)}^{*}(v)=1-\Theta\left(B_{l, l^{\prime}}^{*} / u-|v|\right)
$$

respectively, where $Q_{l, l^{\prime}}$ and $B_{l, l^{\prime}}$ are defined in Eqs. (A22) and

$$
Q_{l, l^{\prime}}^{*}=K_{l, l^{\prime}}^{(*)}\left(q_{* c\left(l, l^{\prime}\right)}\right), \quad B_{l, l^{\prime}}^{*} / u=S_{l, l^{\prime}}^{(*)}\left(q_{* s\left(l, l^{\prime}\right)}\right)
$$

respectively. In Eqs. (A22) and (B13)-(B15) we have used the values (22) and (B7)-(B9). For both the states (18) and (B10) the limits of integration of Eqs. (A33) and (A34) are given by

$$
K_{l, l^{\prime}}^{(0)}\left( \pm q_{c\left(l, l^{\prime}\right)}\right)=K_{l, l^{\prime}}^{(*)}\left( \pm q_{c\left(l, l^{\prime}\right)}\right)= \pm \pi, \quad S_{l, l^{\prime}}^{(0)}\left( \pm q_{s\left(l, l^{\prime}\right)}\right)=S_{l, l^{\prime}}^{(*)}\left( \pm q_{s\left(l, l^{\prime}\right)}\right)= \pm \infty
$$




\section{APPENDIX C: STATE OF MINIMAL ENERGY OF A FAMILY OF STATES WITH COMMON ETA SPIN AND SPIN}

In this Appendix we show that at fixed and finite values of the chemical potential and magnetic field the lowest-energy Hamiltonian eigenstate of a family of states $\left|\eta, S ; \eta_{z}, S_{z}\right\rangle$ with common values $\eta$ and $S$ but different eigenvalues $\eta_{z}$ and $S_{z}$ is the [LWS,LWS], [LWS,HWS], [HWS,LWS], or [HWS,HWS] corresponding to the $\left(l, l^{\prime}\right)$ sector choosed by the signs of the chemical potential and magnetic field.

Obviously, we have that

$$
\hat{H}_{S O(4)}\left|\eta, S ; \eta_{z}, S_{z}\right\rangle=E_{S O(4)}(\eta, S)\left|\eta, S ; \eta_{z}, S_{z}\right\rangle
$$

where $E_{S O(4)}(\eta, S)$ is the eigenenergy of the whole family of states of eta spin $\eta$ and spin $S$ corresponding to the Hamiltonian (2). Moreover, the eigenenergy of $\left|\eta, S ; \eta_{z}, S_{z}\right\rangle$ relative to the Hamiltonian (1) is

$$
E\left(\eta_{z}, S_{z}\right)=E_{S O(4)}(\eta, S)+2 \mu \eta_{z}+2 \mu_{0} H S_{z}
$$

whereas the eigenenergy of the four above LWS's and (or) HWS's is

$$
\begin{aligned}
& E_{[L W S, L W S]}=E_{S O(4)}(\eta, S)-2 \mu \eta-2 \mu_{0} H S, \\
& E_{[L W S, H W S]}=E_{S O(4)}(\eta, S)-2 \mu \eta+2 \mu_{0} H S, \\
& E_{[H W S, L W S]}=E_{S O(4)}(\eta, S)+2 \mu \eta-2 \mu_{0} H S,
\end{aligned}
$$

and

$$
E_{[H W S, H W S]}=E_{S O(4)}(\eta, S)+2 \mu \eta+2 \mu_{0} H S .
$$

For $\eta_{z} \neq \pm \eta$ and (or) $S_{z} \neq \pm S$ out of the four energies

$$
E\left(\eta_{z}, S_{z}\right)-E_{[L W S, L W S]}=2 \mu\left(\eta_{z}+\eta\right)+2 \mu_{0} H\left(S_{z}+S\right)
$$




$$
\begin{aligned}
& E\left(\eta_{z}, S_{z}\right)-E_{[L W S, H W S]}=2 \mu\left(\eta_{z}+\eta\right)+2 \mu_{0} H\left(S_{z}-S\right) \\
& E\left(\eta_{z}, S_{z}\right)-E_{[H W S, L W S]}=2 \mu\left(\eta_{z}-\eta\right)+2 \mu_{0} H\left(S_{z}+S\right),
\end{aligned}
$$

and

$$
E\left(\eta_{z}, S_{z}\right)-E_{[H W S, H W S]}=2 \mu\left(\eta_{z}-\eta\right)+2 \mu_{0} H\left(S_{z}-S\right)
$$

the energy difference corresponding to the state $|\eta, S ;-\eta,-S\rangle,|\eta, S ;-\eta, S\rangle,|\eta, S ; \eta,-S\rangle$, or $|\eta, S ; \eta, S\rangle$ which belongs to the $\left(l, l^{\prime}\right)$ sector choosed by the signs of the chemical potential $\mu$ and magnetic field $H$ is positive and maximal. This follows directly from the form of the four energies (C7)-(C10).

For example, consider that $\mu>0$ and $H>0$. In this case the eta-spin and spin projections are such that $\eta_{z}<0$ and $S_{z}<0$ [see Eq. (29)], respectively, and we are in the $(-1,-1)$ sector of symmetry $U(1) \otimes U(1)$. It follows then from Eqs. (C7)-(C10) that the [LWS,LWS] Hamiltonian eigenstate $|\eta, S ;-\eta,-S\rangle$ has minimal energy and the gap $E\left(\eta_{z}, S_{z}\right)-E_{[L W S, L W S]}$ is positive when $\eta_{z} \neq-\eta$ and (or) $S_{z} \neq-S$. (Also the energies $E_{[L W S, H W S]}-E_{[L W S, L W S]}, E_{[H W S, L W S]}-E_{[L W S, L W S]}$, and $E_{[H W S, H W S]}-E_{[L W S, L W S]}$ are in this case positive.) There are two minimal gaps, $\Delta_{\eta}$ and $\Delta_{S}$, which correspond to eta spin and spin, respectively. They refer to the states $|\eta, S ;-\eta+1,-S\rangle$ and $|\eta, S ;-\eta,-S+1\rangle$, respectively. Following Eq. (C7) they read $\Delta_{\eta}=2 \mu$ and $\Delta_{S}=2 \mu_{0} H$, respectively.

The same applies for other signs of the chemical potential and magnetic field, i.e. for the remaining three $U(1) \otimes U(1)$ sectors. The corresponding LWS and (or) HWS is the state of minimal energy of the family of states $\left|\eta, S ; \eta_{z}, S_{z}\right\rangle$. In the general case, the minimal gaps are given by

$$
\Delta_{\eta}=2|\mu| ; \quad \Delta_{S}=2 \mu_{0}|H|
$$




\section{REFERENCES}

1 This ansatz was introduced for the case of the isotropic Heisenberg chain by H. A. Bethe, Z. Phys. 71, 205 (1931).

${ }^{2}$ For one of the first generalizations of the Bethe ansatz to multicomponent systems see C. N. Yang, Phys. Rev. Lett. 19, 1312 (1967).

${ }^{3}$ Elliott H. Lieb and F. Y. Wu, Phys. Rev. Lett. 20, 1445 (1968).

${ }^{4}$ For a modern and comprehensive discussion of these issues, see V. E. Korepin, N. M. Bogoliubov, and A. G. Izergin, Quantum Inverse Scattering Method and Correlation Functions (Cambridge University Press, 1993).

${ }^{5}$ F. D. M. Haldane, J. Phys. C 14, 2585 (1981).

${ }^{6}$ Philip W. Anderson, Phys. Rev. Lett. 64, 1839 (1990); 652306 (1990); P. W. Anderson and Y. Ren, in High Temperature Superconductivity, edited by K. S. Bedell, D. E. Meltzer, D. Pines, and J. R. Schrieffer (Addison-Wesley, Reading, MA, 1990).

7 A.G. Izergin, V.E. Korepin, and N. Yu Reshetikhin, J. Phys. A: Math. Gen. 22, 2615 (1989).

${ }^{8}$ Holger Frahm and V. E. Korepin, Phys. Rev. B 42, 10553 (1990); 43, 5653 (1991).

9 J. M. P. Carmelo and P. Horsch, Phys. Rev. Lett. 68, 871 (1992).

10 J. M. P. Carmelo, P. Horsch, and A. A. Ovchinnikov, Phys. Rev. B 46, 14728 (1992).

11 J. M. P. Carmelo and A. H. Castro Neto, Phys. Rev. Lett. 70, 1904 (1993).

12 J. M. P. Carmelo, P. Horsch, D. K. Campbell, and A. H. Castro Neto, Phys. Rev. B 48, $4200(1993)$.

13 J. M. P. Carmelo, A. H. Castro Neto, and D. K. Campbell, Phys. Rev. B 50, 3667 (1994); 3683 (1994); in The physics and mathematical physics of the Hubbard model, edited by D. 
K. Campbell, D. Baeriswyl, J. M. P. Carmelo, F. Guinea, and E. Louis (Plenum, New York, 1994).

${ }^{14}$ J. M. P. Carmelo, A. H. Castro Neto, and D. K. Campbell, Phys. Rev. Lett. 73, 926 (1994).

15 J. Carmelo and A. A. Ovchinnikov, J. Phys.: Condens. Matter 3, 757 (1991).

${ }^{16}$ H. Shiba, Phys. Rev. B 6, 930 (1972).

17 J. Carmelo, P. Horsch, P.-A. Bares, and A. A. Ovchinnikov, Phys. Rev. B 44, 9967 (1991).

18 J. M. P. Carmelo, P. Horsch, and A. A. Ovchinnikov, Phys. Rev. B 45, 7899 (1992).

${ }^{19}$ A. H. Castro Neto, H. Q. Lin, Y.-H. Chen, and J. M. P. Carmelo, to appear in Phys. Rev. B (1994).

${ }^{20}$ J. M. P. Carmelo and N. M. R. Peres, to appear in Phys. Rev. B (1995).

${ }^{21}$ Fabian H. L. Essler and Vladimir E. Korepin, Phys. Rev. Lett. 72, 908 (1994).

${ }^{22}$ C. N. Yang, Phys. Rev. Lett. 63, 2144 (1989).

${ }^{23}$ Fabian H. L. Essler, Vladimir E. Korepin, and Kareljan Schoutens, Phys. Rev. Lett. 67, 3848 (1991); Nucl. Phys. B 372, 559 (1992).

${ }^{24}$ O. J. Heilmann and E. H. Lieb, Ann. N. Y. Acad. Sci. 172, 583 (1971).

${ }^{25}$ E. H. Lieb, Phys. Rev. Lett. 62, 1201 (1989).

${ }^{26}$ C. N. Yang and S. C. Zhang, Mod. Phys. Lett. B 4, 759 (1990).

${ }^{27}$ Stellan Östlund, Phys. Rev. Lett. 69, 1695 (1992).

${ }^{28}$ J. M. P. Carmelo and N. M. R. Peres, unpublished (1994).

${ }^{29}$ C. N. Yang and C. P. Yang, J. Math. Phys. 10, 1115 (1969).

${ }^{30}$ D. Pines and P. Nozières, in The Theory of Quantum Liquids, (Addison-Wesley, Redwood 
City, 1989), Vol. I.

${ }^{31}$ Gordon Baym and Christopher J. Pethick, in Landau Fermi-Liquid Theory Concepts and Applications, (John Wiley \& Sons, New York, 1991).

32 José Carmelo and Dionys Baeriswyl, Phys. Rev. B 37, 7541 (1988). 


$\begin{array}{lllll} & (-1,-1) & (-1,1) & (1,-1) & (1,1) \\ N_{c\left(l, l^{\prime}\right)} & N & N & 2 N_{a}-N & 2 N_{a}-N \\ N_{c\left(l, l^{\prime}\right)}^{*} & N_{a} & N_{a} & N_{a} & N_{a} \\ N_{s\left(l, l^{\prime}\right)} & N_{\downarrow} & N_{\uparrow} & N_{a}-N_{\uparrow} & N_{a}-N_{\downarrow} \\ N_{s\left(l, l^{\prime}\right)}^{*} & N_{\uparrow} & N_{\downarrow} & N_{a}-N_{\downarrow} & N_{a}-N_{\uparrow} \\ q_{F c\left(l, l^{\prime}\right)} & 2 k_{F} & 2 k_{F} & 2\left[\pi-k_{F}\right] & 2\left[\pi-k_{F}\right] \\ q_{c\left(l, l^{\prime}\right)} & \pi & \pi & \pi & \pi \\ q_{F s\left(l, l^{\prime}\right)} & k_{F \downarrow} & k_{F \uparrow} & \pi-k_{F \uparrow} & \pi-k_{F \downarrow} \\ q_{s\left(l, l^{\prime}\right)} & k_{F \uparrow} & k_{F \downarrow} & \pi-k_{F \downarrow} & \pi-k_{F \uparrow}\end{array}$

TABLE 1 - Values of the numbers $N_{\alpha\left(l, l^{\prime}\right)}$ and $N_{\alpha\left(l, l^{\prime}\right)}^{*}$ and of the pseudo-Fermi points and limits of the pseudo-Brillouin zones $(22)$ in the four $\left(l, l^{\prime}\right)$ sectors of symmetry $U(1) \otimes U(1)$. 